\section{A) Check for updates}

Cite this: Food Funct., 2022, 13, 2475

\title{
Cognitive improvement effect of nervonic acid and essential fatty acids on rats ingesting Acer truncatum Bunge seed oil revealed by lipidomics approach $\uparrow$
}

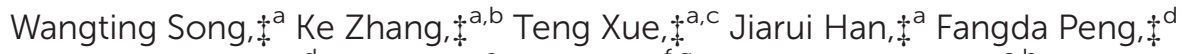

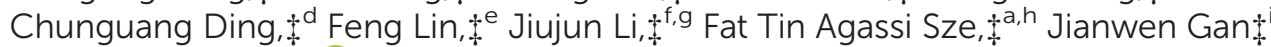 \\ and Xianyang Chen (D)* $*$, c
}

\begin{abstract}
Acer truncatum Bunge seed oil (ASO) is rich in $\omega-9$ (53.93\%) and $\omega-6$ (30.7\%) fatty acids (FAs) and characterized by 3-7\% nervonic acid (NA, C24:1 $\omega-9$ ). Evidence suggests that $\omega-9$ FAs such as NA participate in processes of cognitive improvement; however, their mechanism remains ambiguous. In this study, we investigated the effect of ASO on rat memory and the change in lipid profiling and underlying metabolism. After ASO was administrated to rats for one, three and seven days, their capacity for learning and memory significantly increased via the MWM test. Lipid profiling showed alterations in a wide range of metabolic features after ASO was administrated to the rats, in which sphingolipids (SP) in the serum and glycerophospholipids (GP) in the brain were regulated significantly. The changes in the fatty acids in the serum and brain showed the synergetic effects of NA, EA, OA and DHA, where NA, EA and OA exhibited similar change trends. The enrichment analysis based on KEGG indicated that ASO supplementation evoked the pathways of neurotrophin signaling, glycerophospholipid metabolism and sphingolipid metabolism, which are related to memory and cognition improvement. Among the metabolites with different molecular forms, the biomarkers with $\mathrm{C} 24: 1 \omega-9$ chains exhibited a positive correlation with others both in the serum SP and brain GP. These results suggest the synergistic effects of $\omega-9$ FAs and that their conversion into each other may result in enhanced cognition in rats ingesting Acer truncatum Bunge seed oil.
\end{abstract}

\author{
Received 1st November 2021 \\ Accepted 27th December 2021 \\ DOI: $10.1039 / \mathrm{d} 1 \mathrm{fo} 03671 \mathrm{~h}$ \\ rsc.li/food-function
}

\section{Introduction}

Fatty acids (FAs) are essential nutrients for the growth and normal physiological function in the human body. ${ }^{1}$ The composition of FAs represents a strong correlation with the status of the brain. As the most highly consumed and essential poly-

\footnotetext{
${ }^{a}$ Bao Feng Key Laboratory of Genetics and Metabolism, Beijing, China. E-mail: cxyibcas@163.com; Fax: +8610 85095705; Tel: +86 1085095705 ${ }^{b}$ School of Grassland Science, Beijing Forestry University, Beijing, 100083, China ${ }^{c}$ Zhong Guan Cun Biological and Medical Big Data Center, Beijing, China ${ }^{d}$ National Center for Occupational Safety and Health, NHC, Beijing, 102308, China ${ }^{e}$ Department of Neurology, Sanming First Hospital Affiliated to Fujian Medical University, Sanming, Fujian, China

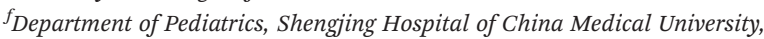
Shenyang, China

${ }^{g}$ Plateau Medical Research Center of China Medical University, Shenyang, China ${ }^{h}$ Graduate Institute of Bioresources, National Pingtung University of Science and Technology, Pingtung, TaiwanChina

${ }^{i}$ Macau University of Science and Technology, Macau, China

$\dagger$ Electronic supplementary information (ESI) available. See DOI: 10.1039/ d1fo03671h

$\$$ All authors contributed equally to this work.
}

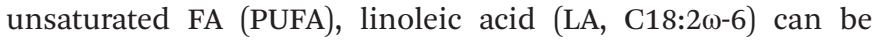
elongated and desaturated to form other bioactive $\omega-6$ PUFAs,

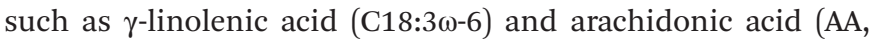

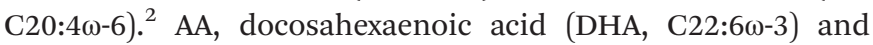
nervonic acid (NA, C24:1 $\omega$-9, $\Delta 15$, cis-15-tetracosenoic acid) are the most important FAs in the nervous system. ${ }^{3}$ AA and DHA contribute to structure formation in neuronal membranes, making up around $20 \%$ dry mass of the brain. ${ }^{4} \mathrm{C} 24: 1 \omega-9$ is the major constituent of the sphingolipids in myelin membranes. ${ }^{5}$ Evidence indicates that $\omega-9$ monounsaturated FAs (MUFAs) are capable of neurotrophic effects. Studies have demonstrated that the intake of an enriched $\omega-9$ MUFA diet is associated with a reduction in anger and irritability. ${ }^{6}$ The most represen-

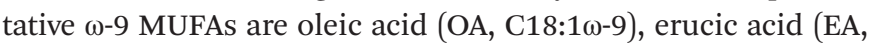

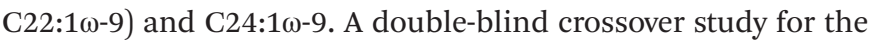
treatment of X-linked adrenoleukodystrophy (X-ALD), a demyelinating brain disorder, with dietary OA and EA as a nutritional supplement showed an increase in white matter following treatment without adverse effects on the diet. ${ }^{7}$

As a particular Acer species in China, Acer truncatum Bunge is an important widespread prominent species in the hard- 
wood forests from north to west China. ${ }^{8}$ Acer truncatum Bunge seed oil (ASO), as a novel edible oil, possesses a proportion of unsaturated FAs that is up to $92 \%$ is richer in LA and OA than other edible oils including sunflower, rapeseed, grape and peanut oil. ${ }^{9,10}$ ASO is an important woody oil, and its nutritional and economic value largely depend on its FA composition. In 2011, ASO was certified as a new food resource by the Ministry of Health of the People's Republic of China. ${ }^{11}$ Especially, ASO is the main plant resource to achieve the largescale production of $\mathrm{C} 24: 1 \omega-9$, whose portion is $3-7 \% .^{12}$

C24:1 $\omega-9$, a very long-chain FA (VLCFA), plays a vital role in human health, especially in brain development. As the main long-chain unsaturated FA in the branch chain of lipids such as phosphatidylcholine (PC), sphingomyelin (SM) and ceramide (Cer), the synthesis of $\mathrm{C} 24: 1 \omega-9$ is the rate-limiting step in the lipid homeostasis of the myelin sheath. ${ }^{13}$ Nerve fibers consist mainly of myelin sheath and axons, and the myelin sheath is surrounded by a thick lipid layer, which determines the speed of saltatory signal conduction. ${ }^{14,15}$ The outer lipid membrane of the myelin sheath is in a dynamic equilibrium of shedding and regeneration, and its production requires various outer membrane lipids as raw materials. Therefore, C24:1 $\omega-9$ is regarded as one of the structural compounds in the nervous system. ${ }^{16}$ Substantial evidence has proven that C24:1 $\omega$-9 deficiency can trigger a series of neurological diseases. A reduction in $\mathrm{C} 24: 1 \omega-9$ has been confirmed to be associated with demyelinating disease, whereas an NAenriched diet can ameliorate ALD. ${ }^{17}$ However, the amount of C24:1 $\omega$-9 synthesized in humans is relatively low, and thus exogenous supplementation may be considered necessary to ameliorate neuropathy in individuals with a deficiency in $\mathrm{C} 24: 1 \omega-9 .{ }^{13}$ One way for the production of $\mathrm{C} 24: 1 \omega-9$ in vivo is via the conversion of other FAs through a series of biochemical reactions; alternatively, direct ingestion is another way by which C24:1 $\omega-9$ is readily available to the body. ${ }^{18}$

C24:1 $\omega$-9 can also be obtained from certain types of oils. Lewkowicz et al. employed a fish oil mixture rich in C24:1 $\omega-9$ (13\%) and $\omega$-3 PUFAs in a human model of maturating oligodendrocyte precursor cells, and found an enhancement in the synthesis of sphingomyelin and inhibition of proinflammatory cytokines and chemokines. ${ }^{13}$ Cook et al. supplemented Lunaria oil, which is rich in C24:1 $\omega-9$ (6.89\%) and erucic acids

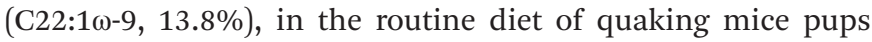
and observed normalization of the level of $\mathrm{C} 24: 1 \omega-9$ in brain sphingomyelin. ${ }^{19}$ ASO is qualified for providing exogenous C24:1 $\omega-9$ as well as other types of essential unsaturated FAs in the human body and represents a new type of plant resource with potential applications for treating human cerebral and neurological problems. ${ }^{20}$ Nevertheless, the effect of ASO supplementation on cognitive function and molecular alterations in the serum and brain is still unclear.

To explore the effect of ASO, a type of novel edible oil, especially on cognitive function, in this study we supplemented ASO to rats for a certain number of days to evaluate its effect on memory enhancement via the Morris water maze (MWM) test. Afterward, we employed lipid profiling to deter- mine the lipid remodeling in the serum and brain. The pathway and multivariate statistical analysis revealed the biological processes and biomarkers related to cognitive function after the supplementation of ASO.

\section{Materials and methods}

\subsection{Animals and treatments}

A total of 30 Sprague-Dawley (SD) rats (180-200 g) was provided by Beijing Vital River Laboratory Animal Technology Co., Ltd. The animals were placed in a room with a temperature of $22{ }^{\circ} \mathrm{C}$ and humidity of $45 \%$, and the animals were exposed to day and night cycles for $12 \mathrm{~h}$ (lighting time 8:00-20:00) and allowed access to food and water ad libitum. The rats were fasted for $12 \mathrm{~h}$ before the experiment and drank freely. The experiment was carried out in strict accordance with the guidelines in the Guide for the Care and Use of Laboratory Animals and ethical standards for experimental animals, which was approved by the Ethics Committee of Vital River Laboratory (Approval No. P2019070).

Thirty six-week-old SD rats were randomly and equally divided into two groups, including the control group and ASO group. Before the formal experiment, the rats were adaptively fed for 1-2 weeks. The ASO group was fed ASO by gavage, and the control group received the vehicle. Based on the individual recommended intake of NA of $0.3 \mathrm{~g} \mathrm{~kg}^{-1} \mathrm{~d}^{-1}$, the equivalent dose of the drug was calculated according to the body weight of a human and the animal, where the daily dose of the rat was equivalent to six times that of adults. After continuous administration, the rats from the control $(n=5)$ and ASO $(n=$ 5) groups were sacrificed via $\mathrm{CO}_{2}$ euthanasia after one, three, and seven days. The weight of the body and brain of each rat were recorded, meanwhile the brain and serum were collected and analyzed by lipidomics (Fig. 1).

\subsection{Morris water maze (MWM)}

The MWM consisted of a circular pool (150 cm diameter) in which mice were trained to escape from the water by swimming to a hidden platform ( $2 \mathrm{~cm}$ beneath the water surface) whose location could only be identified using distal extramaze cues attached to the room walls. ${ }^{21}$ The water temperature was maintained at $24{ }^{\circ} \mathrm{C} \pm 2{ }^{\circ} \mathrm{C}$. The pool was divided into four virtual quadrants (compass locations: NE, NW, SW and SE) by a computerized tracking/image analyzer system (video camcorder coupled to computational tracking system: EthoVision XT 13.0.1216). The platform was placed in the middle of the SE quadrant and remained in the same position throughout the experiment.

According to the general operation of the MWM test, the test in our study included a six-day place navigation test and a spatial probe test on the last day. ${ }^{21,22}$ The navigation training phase consisted of 24 trials: 4 training trials per day and 6 training days with an intertrial interval of $20 \mathrm{~min}$. The rats were released with their heads facing the pool wall from the four compass locations for each training per day and allowed 


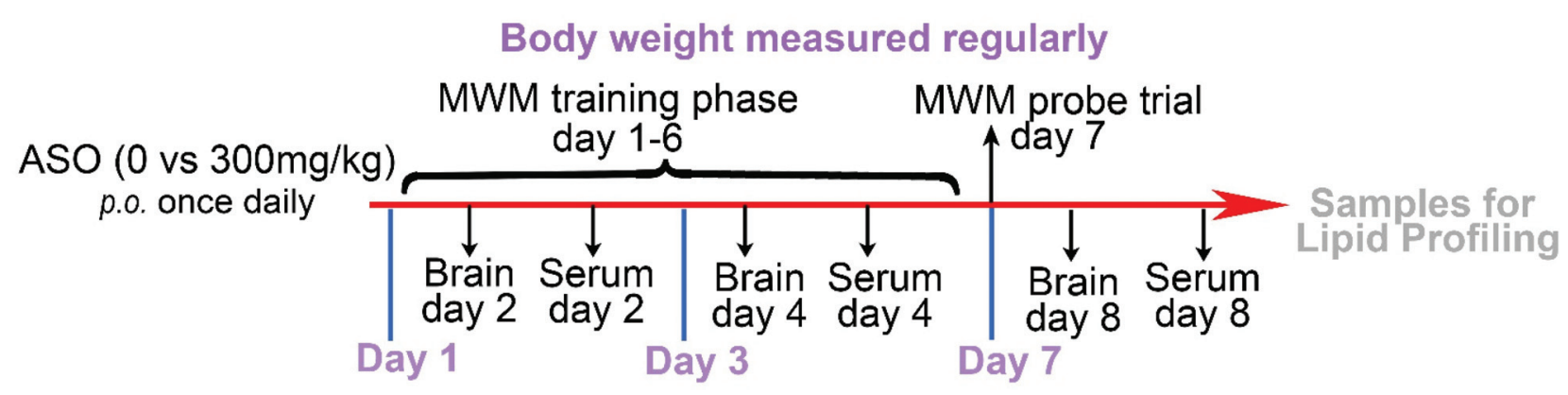

Fig. 1 Dosage regimen and sampling schedule.

to swim and search for the platform within $120 \mathrm{~s}$, and the latency to reach the hidden platform (shown as escape latency) was recorded. If the rats did not locate the platform after 120 $\mathrm{s}$, the animals were manually guided to reach the platform, and the escape latency was recorded as $120 \mathrm{~s}$.

On the 7th day (the day after the navigation training phase), the subjects received a probe trial, in which the platform was removed. The rats were released from the NW start point and allowed to swim freely for $120 \mathrm{~s}$. The path that the rat swam was tracked and analyzed for the proportion of swim time in the target quadrant (SE) of the pool.

\subsection{Chemical reagents}

High-performance liquid chromatography (HPLC)-grade acetonitrile $(\mathrm{ACN})$ and methanol were purchased from Thermo Fisher Scientific Co., Ltd. ASO is a commodity purchased from Baofeng Biotechnology (Beijing) Co., Ltd. The components of ASO were determined by PONY Co., Ltd, Beijing, China, which mainly consisted of FAs, including LA (C18:2 $\omega-6) 30.7 \%$, OA

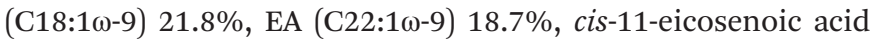

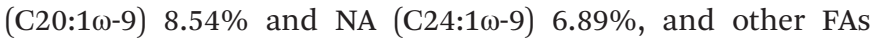
(Table 1 and Table S1†).

Table 1 Ingredient composition of ASO

\begin{tabular}{|c|c|c|}
\hline Ingredient & Description & Content (\%) \\
\hline Linoleic acid & $\mathrm{C} 18: 2 \omega-6$ & 30.7 \\
\hline Oleic acid & C18:1 $\omega-9$ & 21.8 \\
\hline Erucic acid & $\mathrm{C} 22: 1 \omega-9$ & 18.7 \\
\hline cis,cis-11-Eicosenoic acid & $\mathrm{C} 20: 1 \omega-9$ & 8.54 \\
\hline cis-15-Tetracosenoic acid (nervonic acid) & $\mathrm{C} 24: 1 \omega-9$ & 6.89 \\
\hline Palmitic acid & C16:0 & 4.02 \\
\hline Stearic acid & C18:0 & 2.48 \\
\hline$\alpha$-Linolenic acid & $\mathrm{C} 18: 3 \omega-3$ & 1.65 \\
\hline Behenic acid & C22:0 & 0.96 \\
\hline$\gamma$-Linolenic acid & $\mathrm{C} 18: 3 \omega-6$ & 0.74 \\
\hline Tetracosanoic acid & $\mathrm{C} 24: 0$ & 0.42 \\
\hline cis-11,14-Eicosadienoic acid & $\mathrm{C} 20: 2 \omega-6$ & 0.34 \\
\hline Arachidic acid & $\mathrm{C} 20: 0$ & 0.28 \\
\hline cis,cis,cis-11,14,17-Eicosatrienoic acid & $\mathrm{C} 20: 3 \omega-3$ & 0.16 \\
\hline Palmitoleic acid & 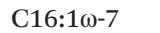 & 0.07 \\
\hline Heptadecanoic acid & $\mathrm{C} 17: 0$ & 0.07 \\
\hline cis-10-Heptadecenoic acid & $\mathrm{C} 17: 1 \omega-7$ & 0.04 \\
\hline Tricosanoic acid & C23:1 & 0.04 \\
\hline cis-13,16-Eicosadienoic acid & $\mathrm{C} 22: 2 \omega-6$ & 0.02 \\
\hline
\end{tabular}

\subsection{Sample preparation for metabolomics analysis}

We adapted previously described methods, where lipids from the serum and brain samples were extracted with isopropanol (IPA). ${ }^{23}$ In brief, $200 \mu \mathrm{L}$ of serum was extracted with $600 \mu \mathrm{L}$ of precooled IPA at a temperature of $4^{\circ} \mathrm{C}$, vortexed for $1 \mathrm{~min}$, and incubated at room temperature for $10 \mathrm{~min}$; subsequently, the extraction mixture was stored overnight at $-20{ }^{\circ} \mathrm{C}$. The samples were centrifuged at $14000 \mathrm{rpm}$ for $20 \mathrm{~min}$, and the supernatants were transferred into a new centrifuge tube and diluted to $1: 10$ with IPA/ACN/ $\mathrm{H}_{2} \mathrm{O}(2.5: 1: 1, \mathrm{v}: \mathrm{v}: \mathrm{v})$. Before $\mathrm{LC} / \mathrm{MS}$ analysis, the sample was stored at $-80^{\circ} \mathrm{C}$. In addition, $50 \mu \mathrm{L}$ was extracted from each serum sample to prepare a quality control (QC) sample.

\subsection{LC-QTOF analysis}

The samples were analyzed using an ACQUITYUPLC (Waters) and XEVO-G2XS quadrupole time-of-flight (QTOF) mass spectrometer (Waters) in ESI mode. ${ }^{24}$ Lipid separation was performed on an Acquity UPLC charged surface hybrid C18 column $(2.1 \times 100 \mathrm{~mm}, 1.7 \mu \mathrm{m}$, Waters $)$, and the gradient mobile phase was composed of $10 \mathrm{mM}$ ammonium formate and $0.1 \%$ formic acid acetonitrile/aqueous solution (A, $60: 40$, $\mathrm{v} / \mathrm{v}$ ) and $10 \mathrm{mM}$ ammonium formate and $0.1 \%$ formic acid isopropanol/acetonitrile solution (B, 90:10, v/v). Before the formal study, pilot experiments including 10-minute, 15-minute, and 20-minute elution periods were carried out to evaluate the potential effects of the mobile phase composition and flow rate on the lipid retention time. We used a 20-minute accelerated elution curve, as described below. The flow rate of the mobile phase was $0.4 \mathrm{~mL} \mathrm{~min}^{-1}$. The column was initially eluted with $40 \% \mathrm{~B}$, graded linearly to $43 \% \mathrm{~B}$ in $2 \mathrm{~min}$, and then increased to $50 \% \mathrm{~B}$ in $0.1 \mathrm{~min}$. In the next $9.9 \mathrm{~min}$, the gradient was further increased to $54 \% \mathrm{~B}$, and then increased to $70 \%$ in $0.1 \mathrm{~min}$. In the last part of the gradient, the amount of B was increased to $99 \%$ in 5.9 min. Finally, solution B returned to $40 \%$ in $0.1 \mathrm{~min}$, and the column was balanced for $1.9 \mathrm{~min}$ before the next injection. The injection volume was $1 \mu \mathrm{L}$. The lipids in both positive and negative modes were detected using a Xevo-G2XS QTOF mass spectrometer, which was operated in MSE mode from $m / z 50-1200$, and the collection time for each scan was $0.2 \mathrm{~s}$. The source temperature was set to $120{ }^{\circ} \mathrm{C}$. The desolvation temperature was $550{ }^{\circ} \mathrm{C}$, the gas 
flow rate was $1000 \mathrm{~L} \mathrm{~h}^{-1}$, and nitrogen was used as the flowing gas. The capillary voltage was $2.0 \mathrm{kV}(+) / 1.5 \mathrm{kV}(-)$, and the cone voltage was $20 \mathrm{~V}$. Leucine encephalin (molecular weight $=$ $555.62 \times 200 \mathrm{pg} \mu \mathrm{L}^{-1}, 1: 1$ acetonitrile : water) was used as the locking mass for accurate mass determination and corrected with $0.5 \mathrm{~mm}$ sodium formate solution. The samples were randomly sorted, and 5 quality control samples were initially injected to adjust the conditions of the column. One QC sample was injected in every 10 samples for analysis to investigate the repeatability of the data.

\subsection{Data preprocessing and analysis}

The original tandem mass spectrometry datasets were generated on a Waters XEVO-G2XS QTOF instrument and processed by the commercial software Progenesis QI 2.0, including raw data import, selection of possible adducts, peak set alignment, peak detection, deconvolution, dataset filtering, noise reduction, compound identification and normalization with some methods. To identify and evaluate significant lipid features between groups, the original data was initially subjected to LOESS regression for normalization, and then $\mathrm{R}$ software version 3.6.3 was employed for univariate and multivariate analyses, pathway analysis, and permutation ANOVA. One-way ANOVA permutation and unpaired Student's $t$-test were conducted to evaluate the significance of the differences among groups, and the Kruskal-Wallis test was used for one-way analysis of variance. Pathway analyses were performed to identify significantly enriched metabolic and signaling pathways in the KEGG database (http://www.genome.jp/kegg).

\section{Results}

\subsection{ASO supplement conferred enhanced memory in rats}

The quality of ASO was strictly controlled in this study. The composition of the fatty acid was determined by gas chromato-

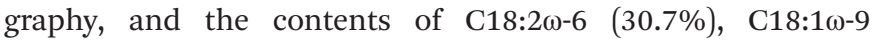

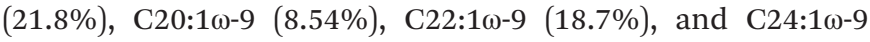
(6.89\%) accounted for $86.63 \%$ of ASO (Table 1). We dosed healthy 6-8-week-old SD rats with $1.8 \mathrm{~g} \mathrm{~kg}^{-1} \mathrm{~d}^{-1}$ ASO supplementation via oral administration for seven consecutive days, while the control group was administrated the equivalent vehicle. There were no adverse effects of ASO administration during the whole experiment. We evaluated synchronously the spatial learning and memory of the rats using the MWM test. On the 1st, 3rd, and 7th day, we recorded the weights of their body and brain, and collected the brain tissue and serum for further analysis.

In the navigation training phase (1st day to 6th day), escape latency to reach the platform in the MWM was recorded, and the time for each rat was calibrated against the time on the 1st training day, which served as a background. A continuous decrease in latency was observed for both the ASO and control groups during the six training days. The latency time of the ASO group was significantly less than the latency time of the control group on the 2nd, 3rd and 6th day (Fig. 2A). As the number of days increased, both the control and ASO rats had significantly shorter platform landing times than on day one. The ASO group started significantly lower on day 2 than on day 1 , and then continued to decline. In contrast, there was a sig-
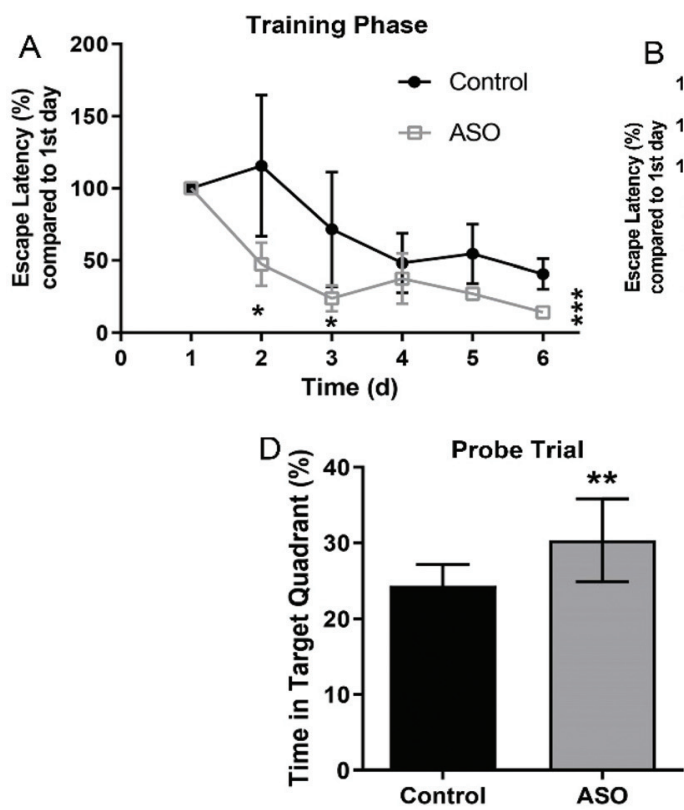
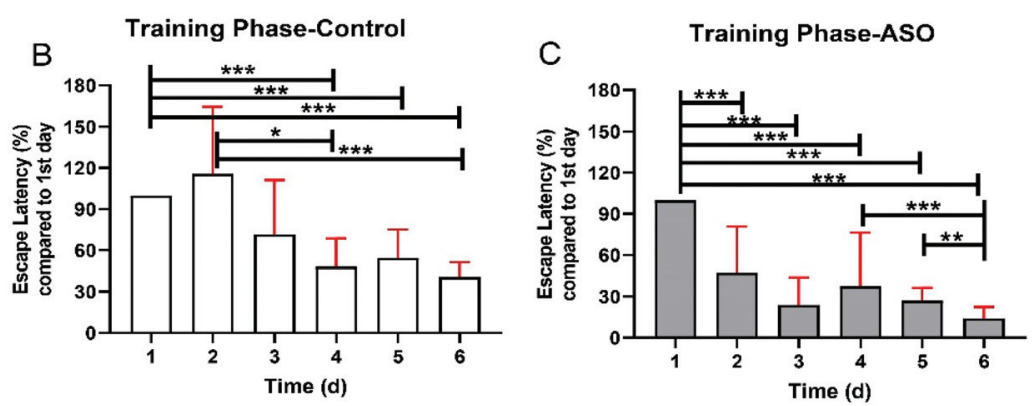

E

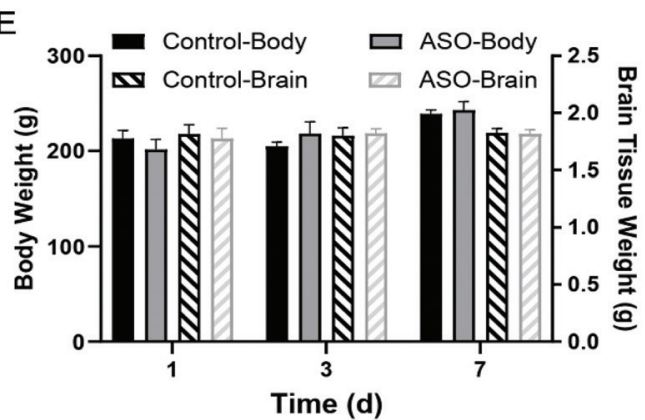

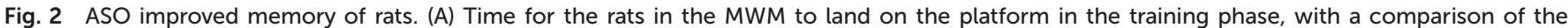

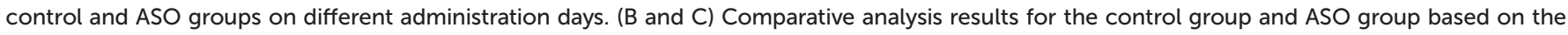

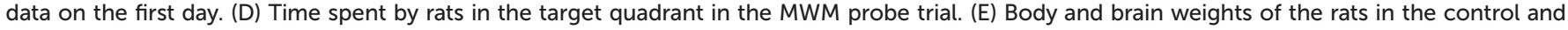
ASO-treated groups. Compared to the control, * indicates $P$-value $<0.05$, **, $P$-value $<0.01$ and ***, and $P$-value $<0.001$. 
nificant difference in the control group from day 4 onwards (Fig. 2B and C). Although the learning ability of the rats on the 6th and 3rd day was better than that of the control group, there was no difference between them. During the probe trial on the 7th day, the time spent in the target quadrant (SE) by the ASO group was $30.3 \%$, which was significantly longer than the time spent in the target quadrant by the control $(24.3 \%)$ (Fig. 2D).

The body weight of the rats increased with time, but there was no significant difference between the control and ASO groups on each day (Fig. 1E). There was no alteration in brain weight during the whole period, and there was no significant difference between the control and ASO groups (Fig. 2E).

\subsection{Profiling of lipid species in serum and brain}

Serum and brain samples from the control and ASO rats were collected for analysis using an untargeted metabolomics approach. Using Progenesis QI 2.0, the lipid features were determined, and 6213 characteristics in the serum and 4786 in the brain were obtained under rigorous quality control. Among the features in the positive ion mode, approximately $85.16 \%$ (2836/3330) and $86.32 \%(2391 / 2770)$ in the serum and brain, respectively, were matched to molecular species with characteristics compatible with lipids or lipid-like compounds. The corresponding distribution in the negative ion mode was $78.01 \%(2249 / 2883)$ and $80.85 \%(1630 / 2016)$ in serum and brain, respectively.

The lipid category for classification was based on the LIPIDMAPS database (http://www.lipidmaps.org). These lipids were categorized into eight classes, including prenol lipids, fatty acyls, sterol lipids, polyketides, glycerolipids, glyceropho- spholipids (GP), sphingolipids (SP) and saccharolipids (SL). There were no marked alterations in the lipids on the 1st day in both the serum and brain. On the 3rd and 7th day, significant and consecutive alterations were observed in the serum SP and brain GP and SL (Fig. S1 $\dagger$ ). The content of SP in the serum continuously accumulated (Fig. 3A). In the brain, the content of GP increased on the 3rd day and decreased on the 7th day (Fig. 3E), contrary to that of SL (Fig. 3I).

Further analysis of the altered lipids in the serum and brain, the lipids from the subclass of SP in the serum and GP and SL in the brain were investigated. The results showed that in the serum, the content of other SP [SP00] was reduced on the 1st day (Fig. 3B). Sphingoid bases [SP01] and neutral glycosphingolipids [SP05] markedly decreased, whereas ceramides [SP02], phosphosphingolipids [SP03], phosphonosphingolipids [SP04] and acidic glycosphingolipids [SP06] significantly increased on the 3rd day (Fig. 3C). Ceramides [SP02], phosphosphingolipids [SP03] and glycosphingolipids [SP06] were all robustly accumulated on the 7th day (Fig. 3D).

In the brain, of the subclass of GP, CDP-glycerols [GP13] notably decreased and glycerophosphocholine [GP16] increased on the 1st day (Fig. 3F), while no significant alteration was found in the subclass of SL (Fig. 3J). On the 3rd day, of the GP subclass, glycerophosphocholines [GP01], glycerophosphoethanolamines [GP02], glycerophosphoinositols [GP06], glycerophosphates [GP10], glycerophosphocholine [GP16] and glycerophosphonoethanolamines [GP17] were prominently augmented (Fig. 3G). In the SL subclass, acylaminosugars [SL01] significantly increased and acyltrehaloses [SL03] decreased (Fig. 3K). On the 7th day, most of the lipids in the GP subclass were significantly reduced, including glyceropho-
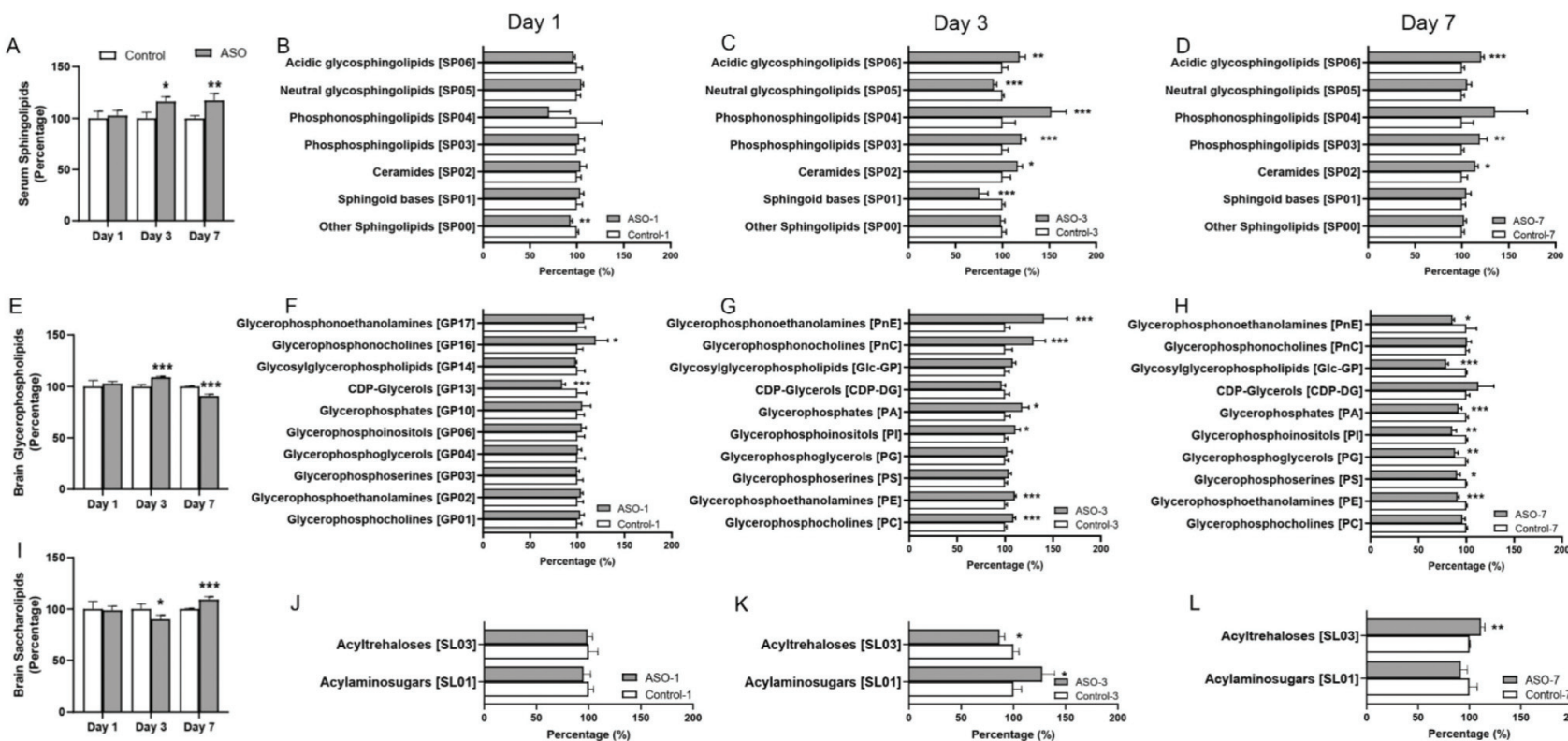

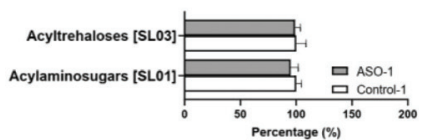

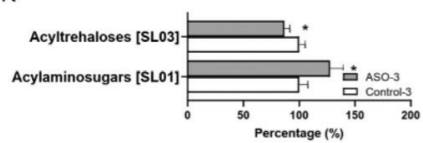

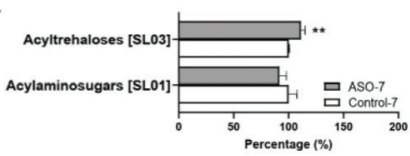

Fig. 3 Changed lipids in the serum and brain after ASO supplementation on the 1st, 3rd and 7th day. Contents of serum SP (A), brain GP (E) and SL (I). The corresponding change in each subclass of lipids on the 1st (B, F and J), 3rd (C, G and K), and 7th (D, H and L) day. Compared to the control, * indicates $P$-value $<0.05,{ }^{* *}, P$-value $<0.01$ and ${ }^{* * *}$, and $P$-value $<0.001$. 
sphoethanolamines [GP02], glycerophosphoinositols [GP06], glycerophosphates [GP10], CDP-glycerols [GP13] and glycerophosphonoethanolamines [GP17] (Fig. 3H). In the subclass of SL, acyltrehaloses [SL03] markedly increased (Fig. 3L).

\subsection{Changes in fatty acid in the serum and brain}

To determine the changes in FAs resulting from ASO supplementation, we selected the top five unsaturated fatty acid chains in ASO (Table 1). From the 1st day to 7th day, all the metabolites containing branch chains of fatty acids of LA

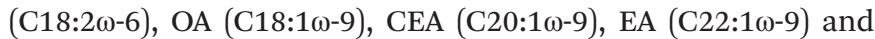
NA (C24:1 $\omega-9)$ were counted. In addition, we displayed the changes in the well-known brain nutrient DHA (C22:6 $\omega-3)$. The overall results showed that only NA was significantly accumulated in the serum and DHA, LA, OA, CEA and NA in the brain significantly increased on the 1st day to 3rd day after ASO supplementation. However, on the 7th day after ASO supplementation, DHA, CEA and NA were continuously accumulated in the serum, while the contents of DHA, LA, OA and NA in the brain decreased (Fig. 4A-F).

Further investigation into the changes in NA, OA, EA and DHA due to ASO supplementation, we analyzed the different molecular forms of these fatty acids, which have been reported to help improve memory. Compared to the control group, the serum results showed that in the lipids of $\mathrm{NA}(\mathrm{C} 24: 1 \omega-9)$, mainly PC(24:1) and PC(P-24:1) were significantly up-regulated. In the lipids of $\mathrm{OA}(\mathrm{C} 18: 1 \omega-9)$, mainly acidic glycosphingolipids (18:1), ceramides(18:1), FAHFA(18:1) and PC(18:1) significantly increased. In the lipids of $\mathrm{EA}(\mathrm{C} 22: 1 \omega-9)$ mainly lysoPC(22:1)

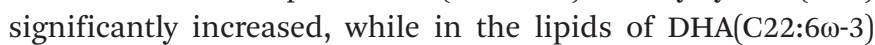
mainly DG(22:6) and TG(22:6) were significantly up-regulated from the 1 st day to the 7 th day (Fig. $5 \mathrm{~A}-\mathrm{D}$ ).

The results showed that in the brain, $\operatorname{lysoPC}(24: 1)$ and PE-

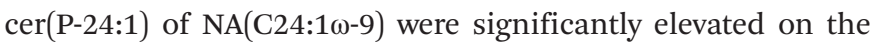

3rd day, but on the 7th day, $\mathrm{PC}(24: 1)$ significantly increased and lysoPE (24:1) significantly decreased. In the lipids of OA

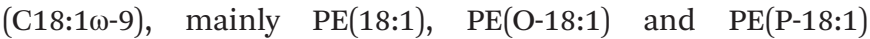
increased significantly on the 1st day and 3rd day, while on the 7th day, acidic lipids(18:1), lysoPC (18:1), LysoPE(18:1) and PC(18:1) significantly decreased, and CDP-DG(18:1), DGTA (18:1), neutral glycosphingolipids(18:1) significantly increased.

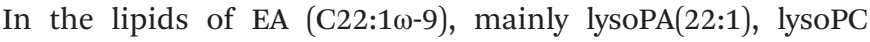
(22:1), $\mathrm{PA}(\mathrm{P}-22: 1)$ and $\mathrm{TG}(22: 1)$ increased significantly from the 1st to 7 th day, while on the 7th day, lysoPC(22:1) decreased. In

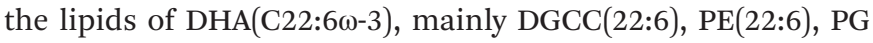
(22:6) and $\mathrm{TG}(22: 6)$ were significantly up-regulated from the 3rd to 7th day, while lysoPE(22:6) and lysoPS(22:6) significantly decreased on the 7th day (Fig. 5E-H). The heat map of clustering showed that the change trends of NA, EA and OA at different time points in the ASO group were similar (Fig. 5A-C and $E-G$ ), while the changes in DHA in the ASO group at different time points were similar to the corresponding control group (Fig. 5D and $\mathrm{H}$ ). We speculated that although supplementation of ASO could cause changes in DHA in the serum and brain, this was likely to be time dependent.

We used a ternary diagram to observe the variation in four fatty acids in three days as three dimensions of their characteristics and investigate their overall variation trends. All the molecular forms of these four fatty acids are shown in a ternary diagram over three days. The closer they are, the more similar they are, and vice versa. Regardless of the serum or

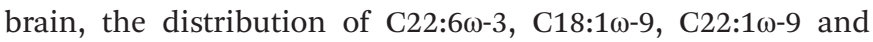
C24:1 $\omega-9$ was concentrated and close, while the plot showed a loose distribution of $\mathrm{C} 22: 6 \omega-3$ (Fig. 5I and J).

\subsection{Feature selection from the serum and brain samples and pathway analysis}

To discover different lipid molecules between the control and ASO groups, a multivariate statistical analysis based on orthog-
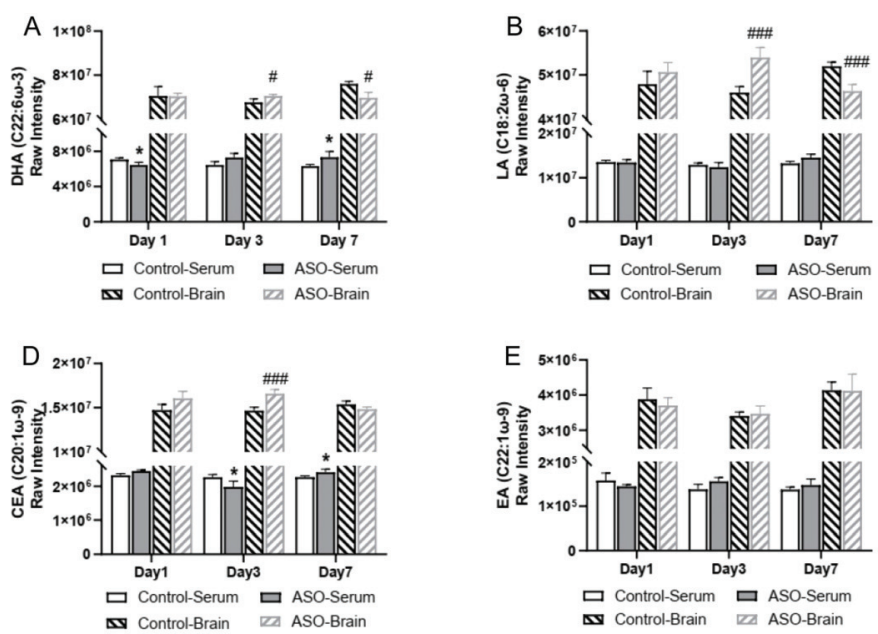

Fig. 4 Content changes of FAs including DHA, LA, OA, CEA, EA and NA in the serum and brain after ASO administration for the 1st, 3rd and 7th day.

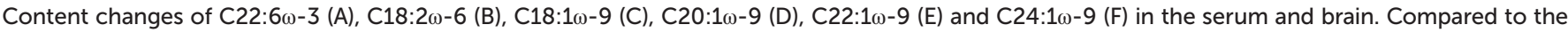
control, * or \# indicates $P$-value $<0.05$, *** or \#\#\#, and $P$-value $<0.001$. * shows the change in the serum and \# in the brain. 

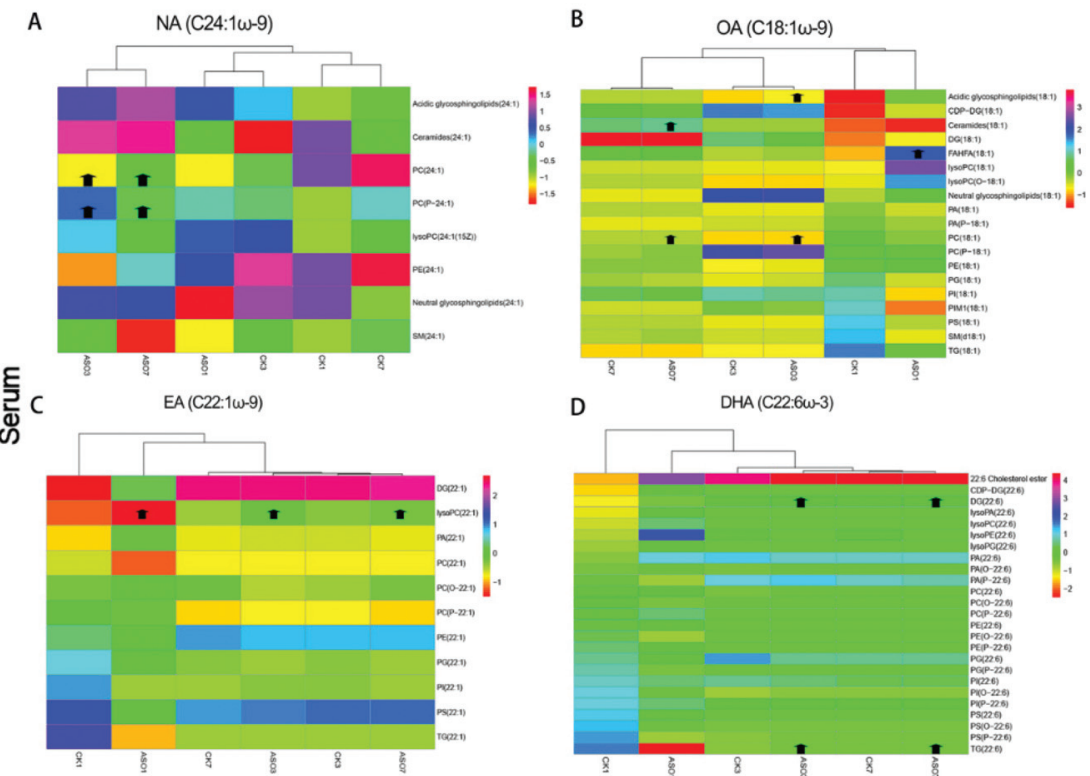

E
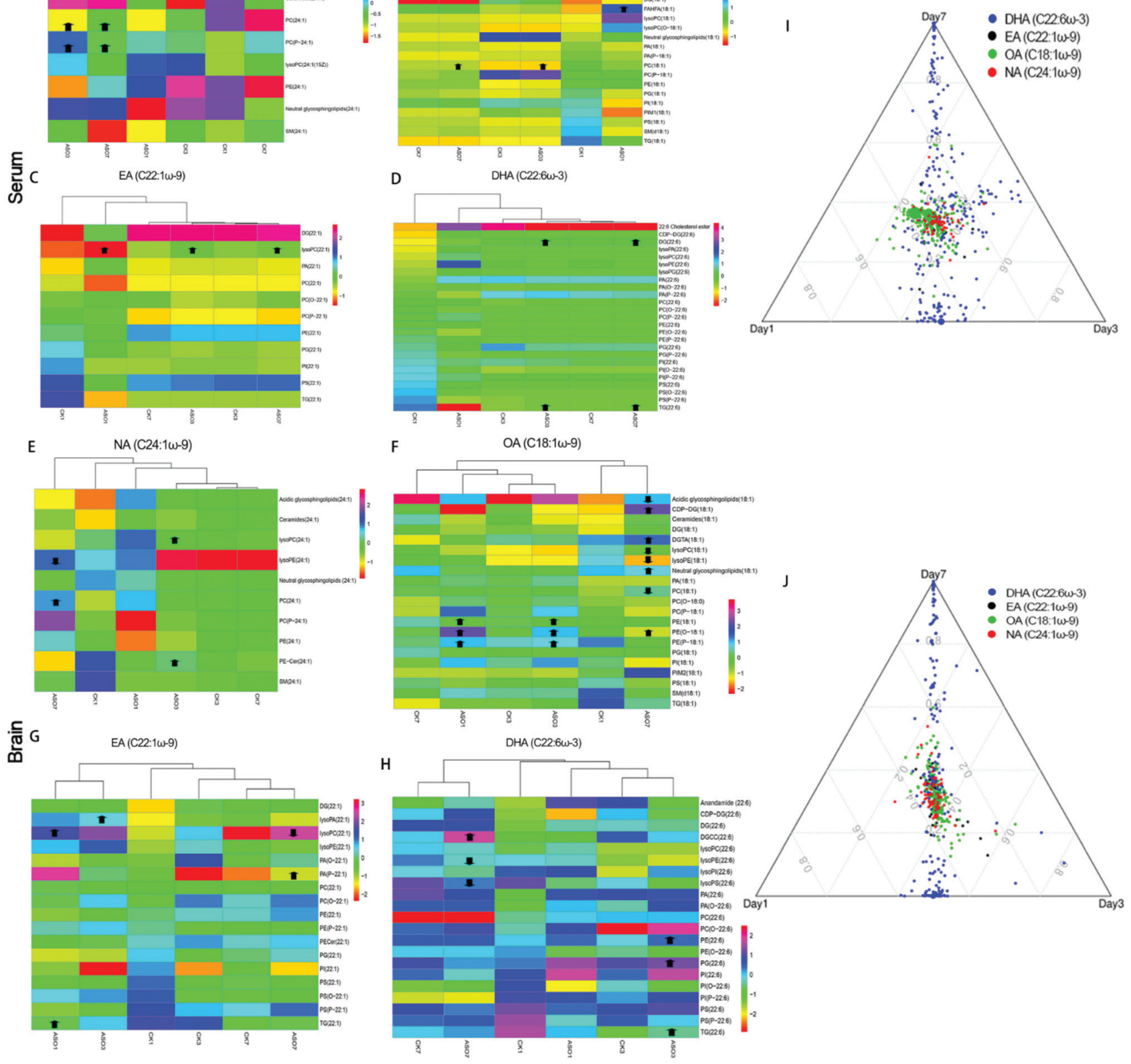

Fig. 5 Changes in the different molecular forms of NA, OA, EA and DHA in the serum and brain. Cluster heat map of the changes in the different molecular forms of NA, OA, EA and DHA in the serum (A-D) and brain (E-H). Compared to the control, arrows indicate significant increase or decrease. Ternary plot by metabolites with $C 22: 6 \omega-3, C 22: 1 \omega-9, C 18: 1 \omega-9$ and $C 24: 1 \omega-9$ in the serum (I) and brain (J), and dot size indicates the raw intensity of each metabolite. ASO1, ASO3 and ASO7 mean ASO administration for 1st, 3rd and 7th day.

onal partial least squares-discriminatory analysis (OPLS-DA) was performed. S-Plots derived from the OPLS-DA model and volcano plots were employed to generate a list of features that were important for group discrimination. To quantify the different features between the control and ASO groups, all detected features were evaluated by the criteria of $P$-value $<$ 0.05 and variable importance projection (VIP) of $>1.0$. In the serum, among the 5056 different metabolites, 56 metabolites were significantly different on the 1 st day (Fig. 6A-C), 90 metabolites were significantly different on the 3rd day (Fig. 6D-F), and 32 candidates were significantly different on the 7th day (Fig. 6G-I). In the brain, among the 4021 different metabolites, 33 metabolites were significantly different on the 1st day (Fig. S1A-C†), 108 metabolites were significantly different on the 3rd day (Fig. S1D-F广), and 109 metabolites were significantly different on the 7th day (Fig. S1G-I†). 

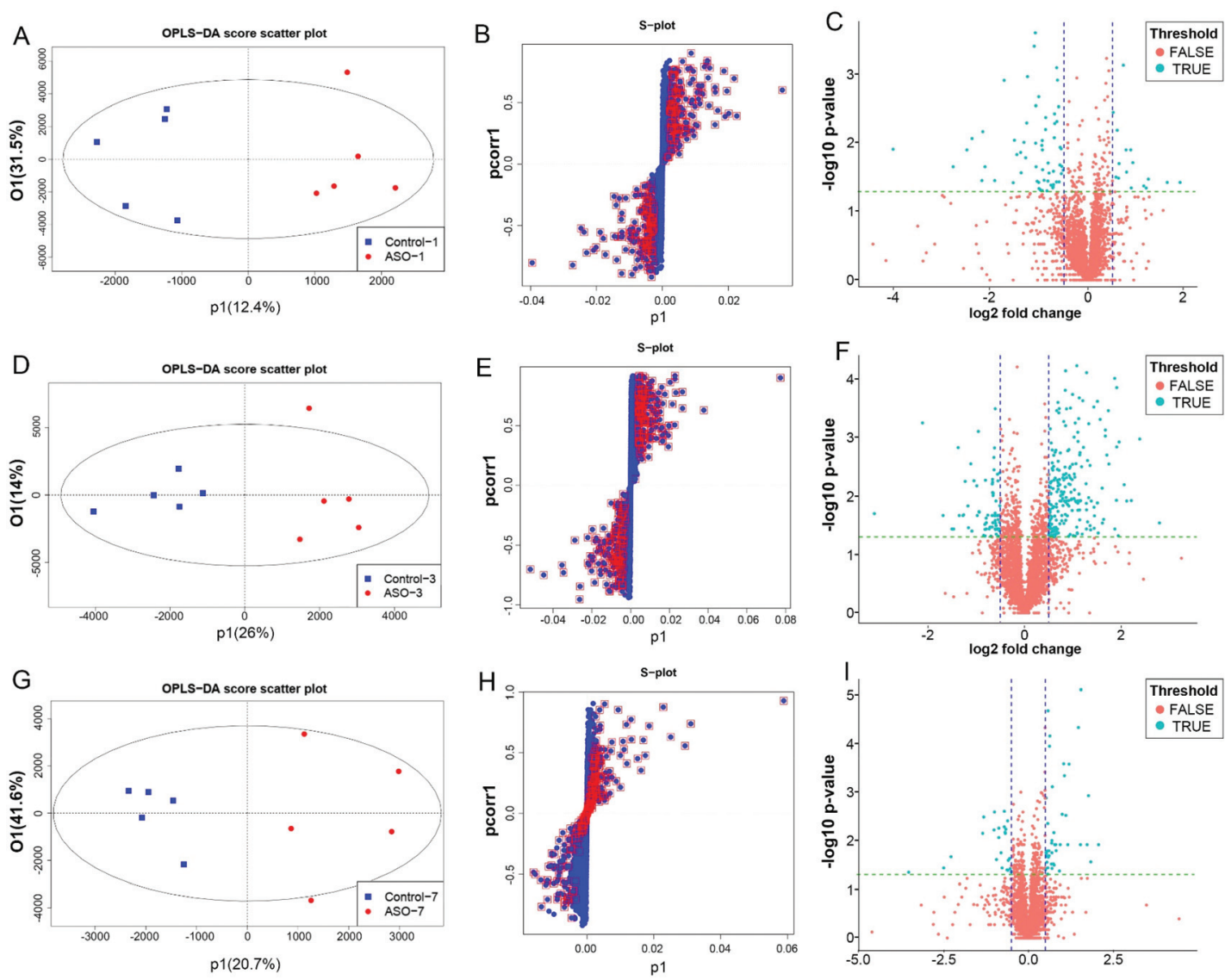

Fig. 6 Feature selection of serum based on multivariate analysis of ASO supplementation on the 1st (A-C), 3rd (D-F) and 7th (G-I) day. (A, D and G) OPLS-DA score scatter plot of serum lipidomics. (B, E and H) S-plot for serum metabolites selection based on covariant and correlation. p1: covariance of projection-based OPLS-DA model and pcorr1: correlation of projection-based OPLS-DA model. (C, F and I) Volcano plots showing both the $P$-value and fold change of metabolites. In the volcano plots, TRUE (blue) represents the metabolite that matched the filter criteria, and FALSE (red) represents the metabolite that did not match the filter criteria.

The serum and brain pathway enrichment analysis based on the Kyoto Encyclopedia of Genes and Genomes (KEGG) is shown in Tables 2 and 3 (with Holm $P$-value $<0.1$ ). Combined with the results of the Venn diagram (Fig. S3†), it can be seen that the supplementation of ASO interfered with glycerol phospholipid metabolism, glycerol metabolism and fat digestion and absorption in the serum from the 1st to 7 th day. With an increase in the time of ASO intake, four more pathways were induced from the 3rd to 7 th day, including the neurotrophin signaling pathway, serotonergic synapse, sphingolipid metabolism and steroid biosynthesis. Besides, the phospholipase D signaling pathway on the 1st day and oxidative phosphorylation and arachidonic acid metabolism on the 7th day were mediated in the serum (Table 2). As for the brain, ASO intake persistently mediated eight pathways including neurotrophin signaling pathway, glycerophospholipid metabolism, sphingolipid metabolism, fat digestion and absorption, thermogenesis, phospholipase D signaling pathway, glycerolipid metabolism and serotonergic synapse. Besides, the phosphatidylinositol signaling system was mediated on the 1st day and AMPK signaling pathway on the 7th day (Table 3).

\subsection{Correlation analysis of metabolites and C24:1-lipid biomarkers in serum and brain}

Supplementation with ASO induced alterations in a wide range of metabolic features. Thus, to better understand the relationship between the metabolites in the serum and brain, we screened the candidates from the metabolites with twelve unsaturated FA chains from the SP lipids in the serum and GP lipids in the brain as biomarkers. On the 1st day, there were no candidates with unsaturated FA chains in the serum SP lipids, and eight candidates were screened from the brain GP lipids, of which the FA chains were concluded to be $\mathrm{C} 18: 3 \omega-3$ (PS), C22:5 $\omega-3$ (PC),

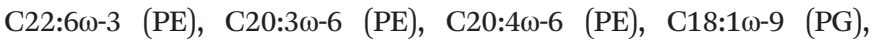
C20:1 $\omega-9$ (PC) and C22:1 $\omega-9$ (PC) (Tables S2 and S3†).

On the 3rd day, $\operatorname{SM}(\mathrm{d} 17: 1 / 24: 1)$ and $\operatorname{Cer}(\mathrm{d} 18: 1 / 24: 1(15 Z))$ were detected in the serum SP lipids, all concluded to be $\mathrm{C} 24: 1 \omega-9$. There were 41 candidates in the brain GP lipids, which were concluded to be C18:3 $\omega-3$ (PC, PE), C20:5 $\omega-3$ (PE),

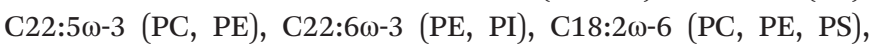

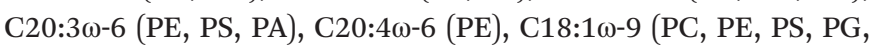

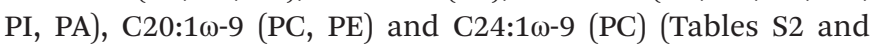


Table 2 KEGG pathway enrichment in the serum

\begin{tabular}{|c|c|c|c|c|c|c|}
\hline Pathway name & Match status & $p$ & Holm $P$ & FDR & HitsName & Time \\
\hline Glycerolipid metabolism & $4 / 38$ & $1.52 \times 10^{-7}$ & $1.2 \times 10^{-5}$ & $6.2 \times 10^{-7}$ & $\mathrm{C} 00681|\mathrm{C} 00422| \mathrm{C} 00162 \mid \mathrm{C} 02133$ & 1st day \\
\hline Fat digestion and absorption & $4 / 13$ & $9.5 \times 10^{-10}$ & $8.74 \times 10^{-8}$ & $6.78 \times 10^{-8}$ & C00162|C01885|C00165|C00422 & 3rd day \\
\hline Neurotrophin signaling pathway & $2 / 5$ & $6.69 \times 10^{-7}$ & $5.75 \times 10^{-5}$ & $8.79 \times 10^{-6}$ & C00165|C00195 & 3rd day \\
\hline Glycerolipid metabolism & $3 / 38$ & $1.59 \times 10^{-5}$ & 0.001303 & 0.000117 & $\mathrm{C} 00162|\mathrm{C} 01885| \mathrm{C} 00422$ & 3rd day \\
\hline Serotonergic synapse & $1 / 42$ & 0.013834 & 0.055341 & 0.014142 & C00165 & 3rd day \\
\hline Steroid biosynthesis & $1 / 58$ & 0.025513 & 0.055341 & 0.025513 & C01694 & 3rd day \\
\hline Neurotrophin signaling pathway & $2 / 5$ & $2.43 \times 10^{-7}$ & $2.16 \times 10^{-5}$ & $1.19 \times 10^{-5}$ & C00165|C00195 & 7th day \\
\hline Fat digestion and absorption & $2 / 13$ & $6.85 \times 10^{-6}$ & 0.000576 & 0.000102 & C00422|C00165 & 7th day \\
\hline Glycerophospholipid metabolism & $3 / 56$ & $1.92 \times 10^{-5}$ & 0.001551 & 0.000189 & C00670|C03819|C01233 & 7th day \\
\hline Serotonergic synapse & $2 / 42$ & 0.000261 & 0.013413 & 0.000541 & C00165|C00909 & 7th day \\
\hline Oxidative phosphorylation & $1 / 16$ & 0.00109 & 0.019909 & 0.001311 & C00399 & 7th day \\
\hline
\end{tabular}

Table 3 KEGG pathway enrichment in the brain

\begin{tabular}{|c|c|c|c|c|c|c|}
\hline Pathway name & Match status & $p$ & Holm $P$ & FDR & HitsName & Time \\
\hline Neurotrophin signaling pathway & $2 / 5$ & $4.21 \times 10^{-7}$ & $3.62 \times 10^{-5}$ & $1.67 \times 10^{-5}$ & C00165|C00195 & 1st day \\
\hline Glycerophospholipid metabolism & $4 / 56$ & $1.11 \times 10^{-6}$ & $9.37 \times 10^{-5}$ & $2.16 \times 10^{-5}$ & C00670|C01233|C03819|C00269 & 1st day \\
\hline Sphingolipid metabolism & $3 / 25$ & $1.49 \times 10^{-6}$ & 0.000122 & $2.16 \times 10^{-5}$ & $\mathrm{C} 12145|\mathrm{C} 00195| \mathrm{C} 12126$ & 1st day \\
\hline Fat digestion and absorption & $2 / 13$ & $1.18 \times 10^{-5}$ & 0.000934 & 0.000114 & $\mathrm{C} 00165 \mid \mathrm{C} 00422$ & 1st day \\
\hline Thermogenesis & $2 / 23$ & $7.16 \times 10^{-5}$ & 0.005374 & 0.000327 & $\mathrm{C} 00165 \mid \mathrm{C} 00422$ & 1st day \\
\hline Phospholipase D signaling pathway & $1 / 11$ & 0.000712 & 0.024218 & 0.001068 & $\mathrm{C} 00165$ & 1st day \\
\hline Phosphatidylinositol signaling system & $1 / 29$ & 0.00506 & 0.037708 & 0.005435 & C00269 & 1st day \\
\hline Glycerolipid metabolism & $1 / 38$ & 0.008595 & 0.037708 & 0.008797 & $\mathrm{C} 00422$ & 1st day \\
\hline Serotonergic synapse & $1 / 42$ & 0.010437 & 0.037708 & 0.010437 & C00165 & 1st day \\
\hline Fat digestion and absorption & $3 / 13$ & $1.64 \times 10^{-7}$ & $1.41 \times 10^{-5}$ & $1.41 \times 10^{-5}$ & $\mathrm{C} 01558|\mathrm{C} 00165| \mathrm{C} 00422$ & 3rd day \\
\hline Neurotrophin signaling pathway & $2 / 5$ & $6.69 \times 10^{-7}$ & $5.69 \times 10^{-5}$ & $2.88 \times 10^{-5}$ & C00195|C00165 & $3 \mathrm{rd}$ day \\
\hline Glycerolipid metabolism & $3 / 38$ & $1.59 \times 10^{-5}$ & 0.001271 & 0.000195 & C02133|C00422|C06041 & 3rd day \\
\hline Glycerophospholipid metabolism & $3 / 56$ & $7.55 \times 10^{-5}$ & 0.00566 & 0.000456 & C01233|C00670|C03819 & 3 rd day \\
\hline Thermogenesis & $2 / 23$ & 0.000113 & 0.007851 & 0.000463 & $\mathrm{C} 00165 \mid \mathrm{C} 00422$ & 3rd day \\
\hline Sphingolipid metabolism & $2 / 25$ & 0.000146 & 0.009488 & 0.000542 & C06062|C00195 & $3 \mathrm{rd}$ day \\
\hline Phospholipase D signaling pathway & $1 / 11$ & 0.000955 & 0.02962 & 0.00137 & $\mathrm{C} 00165$ & 3rd day \\
\hline Serotonergic synapse & $1 / 42$ & 0.013834 & 0.035217 & 0.013834 & C00165 & 3rd day \\
\hline Neurotrophin signaling pathway & $2 / 5$ & $6.69 \times 10^{-7}$ & $5.89 \times 10^{-5}$ & $2.01 \times 10^{-5}$ & C00195|C00165 & 7 th day \\
\hline Glycerolipid metabolism & $3 / 38$ & $1.59 \times 10^{-5}$ & 0.001335 & 0.000169 & C02133|C00162|C06041 & 7th day \\
\hline Fat digestion and absorption & $2 / 13$ & $1.87 \times 10^{-5}$ & 0.001517 & 0.000169 & $\mathrm{C} 00165 \mid \mathrm{C} 00162$ & 7th day \\
\hline Glycerophospholipid metabolism & $3 / 56$ & $7.55 \times 10^{-5}$ & 0.005735 & 0.000415 & $\mathrm{C} 00670|\mathrm{C} 03819| \mathrm{C} 01233$ & 7 th day \\
\hline Thermogenesis & $2 / 23$ & 0.000113 & 0.007957 & 0.000424 & C00165|C00162 & 7th day \\
\hline Phospholipase D signaling pathway & $1 / 11$ & 0.000955 & 0.032487 & 0.00141 & C00165 & 7th day \\
\hline AMPK signaling pathway & $1 / 23$ & 0.004264 & 0.051171 & 0.004858 & C00162 & 7th day \\
\hline Sphingolipid metabolism & $1 / 25$ & 0.005031 & 0.055341 & 0.00559 & C00195 & 7th day \\
\hline Serotonergic synapse & $1 / 42$ & 0.013834 & 0.055341 & 0.01399 & C00165 & 7th day \\
\hline
\end{tabular}

$\mathrm{S} 3 \dagger)$. Most of the candidates in were brain positively correlated with serum $\operatorname{SM}(\mathrm{d} 17: 1 / 24: 1)$ or $\operatorname{Cer}(\mathrm{d} 18: 1 / 24: 1(15 Z))$, especially the significant correlation between cer (d18:1/24:1 (15z)) in the serum and PC $(24: 1(15 z) / 18: 1(9 z))$ in the brain (Fig. 7A).

On the 7 th day, $\operatorname{SM}(\mathrm{d} 17: 1 / 24: 1)$, Cer(d18:1/24:1(15Z)) and GalCer(d18:1/24:1(15Z)) were detected in the serum SP lipids,

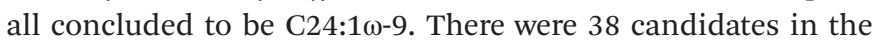
brain GP lipids, which were concluded to be C18:3 $\omega-3$ (PC),

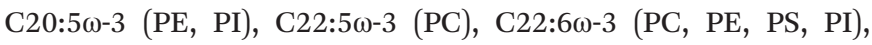

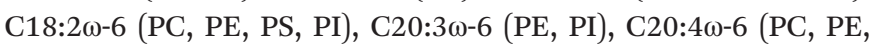

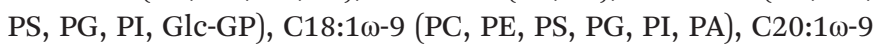

(PE, PA) and C24:1 1 -9 (PC) (Tables S2 and S3†). The candidates in the brain mainly showed a negative correlation with that in the serum on the 7th day. However, $\operatorname{PC}(24: 1(15 Z) / 18: 3$ $(9 Z, 12 Z, 15 Z)), \operatorname{PI}(22: 4(7 Z, 10 Z, 13 Z, 16 Z) / 18: 1(9 Z))$ and PS(P-20:0/ $20: 4(5 Z, 8 Z, 11 Z, 14 Z))$ in the brain were positively correlated with the serum candidates, notably, the correlation between $\operatorname{PC}(24: 1(15 Z) / 18: 3(9 Z, 12 Z, 15 Z))$ in the brain and $\operatorname{SM}(\mathrm{d} 17: 1 /$ 24:1) or GalCer(d18:1/24:1(15Z)) in the serum presented a statistical significance (Fig. 7B).

A total of six screened candidates with C24:1 $\omega^{-9}$ chains in the serum and brain maintained a positive correlation on the 

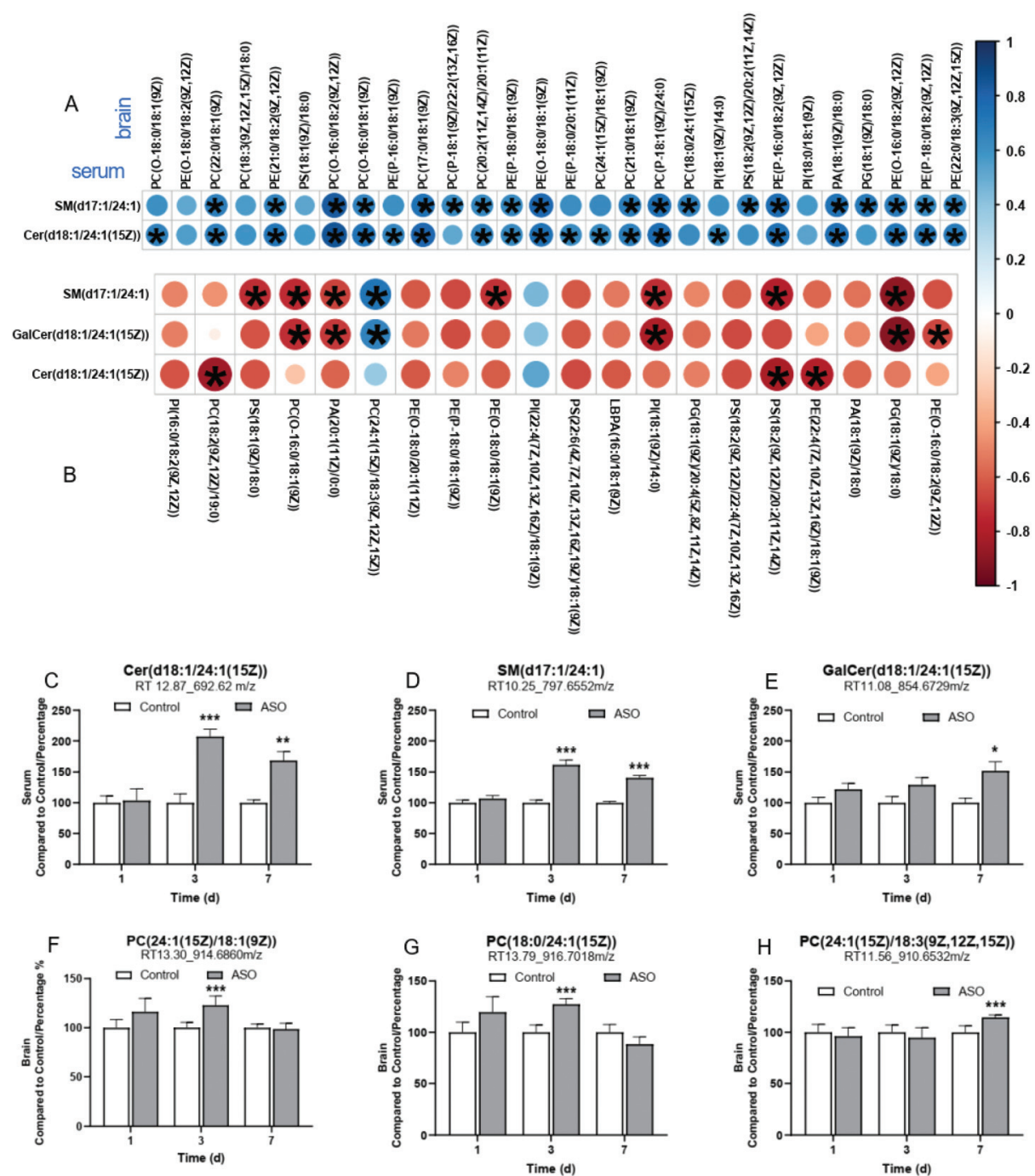

Fig. 7 Correlation analysis between the serum and brain. Correlation analysis of metabolites between the serum and brain on the 3rd (A) and 7th (B) day. * indicates significant differences from the control group at $P$-value $\leq 0.05$. Contents of the biomarkers in the serum (C-E) and brain (F-H) after ASO administration on the 1st, 3rd and 7th day. Compared to the control, * indicates $P$-value $<0.05, * *, P$-value $<0.01$ and $* * *, P$-value $<0.001$.

3rd and 7th day. We arranged these biomarkers and displayed the contents from the 1 st to 7 th day. The contents of the six biomarkers in the serum and brain were all accumulated. In the serum, the contents of $\operatorname{SM}(\mathrm{d} 17: 1 / 24: 1)$ and $\operatorname{Cer}(\mathrm{d} 18: 1 / 24: 1$ $(15 Z))$ were dramatically enhanced from the 3rd to 7 th day (Fig. 7A and B), and GalCer(d18:1/24:1(15Z)) robustly increased on the 7 th day (Fig. 7C). In the brain, the content of $\operatorname{PC}(24: 1$ $(15 Z) / 18: 1(9 Z))$ and PC(18:0/24:1(15Z)) markedly accumulated on the 3rd day (Fig. 7D and E), and $\operatorname{PC}(24: 1(15 Z) / 18: 3$ $(9 Z, 12 Z, 15 Z))$ on the 7 th day (Fig. $7 F)$.

\section{Discussion}

During normal brain development, astrocytes contribute a large number of lipids, which bind to myelin. Importantly, the fatty diet restored the myelin phenotype in conditionally mutated mice. ${ }^{25,26}$ Evidence suggests that dietary lipids can compensate for decreased lipid synthesis in the brain. ${ }^{27}$ As a novel edible oil, ASO is highlighted by the abundance of unsaturated FAs, which mainly consists of $55.9 \%$

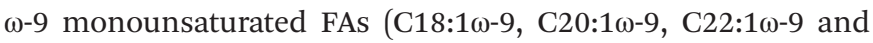
C24:1 $\omega-9$ ) and $30.7 \%$ $\omega-6$ polyunsaturated FAs (Table 1). According to the process of lipid digestion and absorption, dietary lipid intake is directly reflected in a change in blood metabolites, while a change in brain metabolites is an indirect effect. Previous studies have shown that fatty acids such as long-chain fatty acids (LCFAs) exert beneficial effects on white matter microstructural integrity and gray matter volume in the frontal, temporal, parietal, and limbic areas, and on carotid intima media thickness and diastolic blood pressure. ${ }^{28}$ In addition, the nerve fibers in the brain are mainly concentrated in the white matter, and the outer myelin sheath membrane of nerve fibers harbors nearly half of the lipids in the brain. ${ }^{29}$ 
Myelin itself is about $75-80 \%$ lipid by dry weight, with cholesterol, phosphatidylcholine, sphingomyelin, ceramide, glucosyl-ceramide, and sulfatide being the major lipid species. ${ }^{30}$ In adult white matter, nervonic acid with a content of $36 \%$, is also the major FA component of sphingomyelin. ${ }^{31,32}$ Unlike DHA, which is mainly found in the cerebral cortex, the lipids involved in ASO including linoleic acid (LA, C18:2 $\omega-6,30.7 \%$ ),

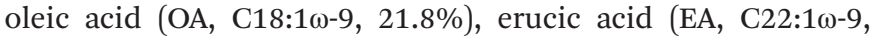

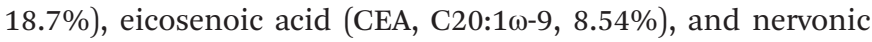

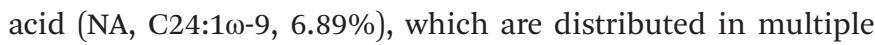
regions of the brain in vivo. In our study, we carried out the experiment adopting the whole brain tissue to reveal the lipid remodeling induced by $\omega-9$ fatty acids, especially NA supplementation, from an overall perspective. To study the behavioral changes in animals due to ASO, the classic Morris water maze test was used to test the learning and memory ability of animals. This experiment was performed for 5-7 days in previous studies, ${ }^{21,22}$ and therefore we chose to conduct a 7 -day feeding experiment to detect the fatty acid metabolism mechanism of ASO. We supplemented ASO to rats and observed a reduction in the time spent by the rats landing the platform in the MWM training phase (Fig. 2D), which represented an improvement in spatial learning. Subsequently, in the probe trial, the rats in the ASO group exhibited a preference for the target quadrant where the platform was located (Fig. 2E), indicating the improvement in memory ability. The MWM experiment results also showed that the learning ability of the rats on the 6th day and 3rd day was superior to that of the control group from the 6-day positioning navigation experiment, but there was no difference between them. Based on the fact that there were no significant differences in FAs between the 3rd and 6th day (Fig. 4), we inferred that the effect on the third day is not better than that on the 7th day.

ASO supplementation resulted in an improvement in the cognitive function of the rats, coupled with lipid remodeling in both their serum and brain. ASO supplementation stimulated the accumulation of SP in the serum (Fig. 3A) and GP and SL in the brain (Fig. 3E and I). GP, SP and SL are commonly used as components of cellular membranes; moreover the GP and SP classes play signaling roles in diverse cellular processes. $^{33}$ Thus, the aberrant metabolism of GP and SP is associated with various neurodegenerative diseases, such as Parkinson's disease, ${ }^{34}$ and also induces depression- and anxiety-related behaviors. ${ }^{35}$ Much evidence suggests that dietary oil, especially long-chain FAs, can synergistically influ-

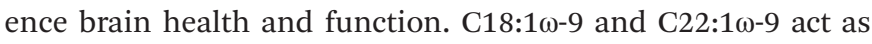
major ingredients of Lorenzo's oil for dietary therapy of X-ALD. ${ }^{36}$ As a neuroactive molecule, C18:1 $\omega$-9 was applied for the treatment of neuropathic pain after neurotrauma, ${ }^{37}$ and C22:1 $\omega$-9 supplementation enhanced memory in a cognitive impairment mouse model. ${ }^{38} \mathrm{C} 24: 1 \omega-9$ is naturally involved as the major constituent of sphingolipids in the myelin membrane $^{5}$ and essential for the growth and maintenance of brain physiology and peripheral nervous tissue. ${ }^{16}$ Multiple studies have demonstrated C24:1 $\omega$-9 deficiency in individuals with neurodegeneration, ${ }^{3,39}$ and $\mathrm{C} 24: 1 \omega-9$ supplementation as a type of nutraceutical is considered to improve brain development and cognition. C18:2 $\omega-6$ is an essential precursor of AA

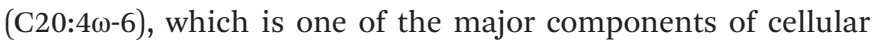
membranes and of special importance to the brain and blood vessels. ${ }^{40} \mathrm{C} 20: 4 \omega-6$ serves as an intercellular messenger in the central nervous system once released from membrane phospholipids. ${ }^{40}$ C18:2 $\omega-6$ deficiency is associated with reduced

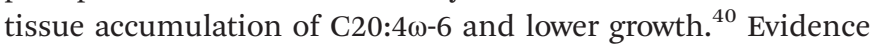

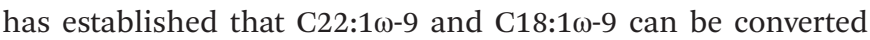
to $\mathrm{C} 24: 1 \omega-9$ in the brain via elongation of their chain in rats. ${ }^{41,42}$ We speculated that the enhanced cognitive ability of the ASO-supplemented rats was attributed to the synergistic effects of NA and essential FAs.

Besides the top 5 unsaturated fatty acids in ASO content, we also observed the well-known DHA, although ASO does not contain it. We found that on the third day, all the substances showed a significant upward trend (Fig. 4), while on the 7th day, all the substances decreased, including DHA and NA (Fig. 5). The changes in the FAs in the ASO group and the control group suggest that ASO regulates lipid remodeling with a faster response, possibly to improve cognitive performance in rats (Fig. 4). According to previous reports, the synthesis of $\omega-9$ fatty acids in the human body is lower than that of other species, ${ }^{43}$ and the intake of exogenous NA can support the remyelination process by improving the sphingolipid synthesis of oligodendrocytes (OLs) ${ }^{44}$ One possibility for the dynamic change in FAs is that the loss of NA may result from its binding via an amide bond to a sphingosine base ${ }^{45}$ during sphingolipid synthesis by finally differentiated OLs. ${ }^{46}$ The other possibility is the downregulation of SCD or FA elongase in oligodendrocytes and/or astrocytes by mediator signaling. Both of these would deplete the supply of DHA (C22:6 $\omega-3)$ and NA (C24:1 $\omega-9)$ for sphingolipid synthesis. The most abundant ingredients in ASO are $\omega-6$ and $\omega-9$ unsaturated FAs, whose content reaches $86.63 \%$ (Table 1). We found that lysoPE(22:6) and lysoPS(22:6) were significantly down-regulated on the 7th day (Fig. 5). According to the unsaturated FA biosynthesis pathway, the excessive intake of $\omega-6$ and $\omega-9$ unsaturated FAs may simultaneously inhibit the biosynthesis of $\omega-3$ unsaturated FAs (Fig. 8A). We inferred that DHA (C22:6 $\omega-3$ ) biosynthesis was affected and lipid metabolism was shifted to NA

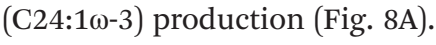

Notably, C24:1 1 -9 was the unique FA chain screened in the serum SP, and simultaneously correlated positively between the serum and brain, and regarded as biomarkers, including Cer(d18:1/24:1(15Z)) SM(d17:1/24:1) and GalCer(d18:1/24:1 $(15 Z))$ in the serum, and $\operatorname{PC}(24: 1(15 Z) / 18: 1), \operatorname{PC}(18: 0 / 24: 1(15 Z))$ and $\operatorname{PC}(24: 1(15 Z) / 18: 3(9 Z, 12 Z, 15 Z))$ in the brain (Fig. 7). Ceramide is a key sphingolipid metabolite in both the biosynthesis and degradation of complex sphingolipids. ${ }^{47} \mathrm{SM}$ and PC serve as structural constituents of cell membranes and plasma lipoproteins. ${ }^{48}$ Galactosylceramide is integral to neural function, performing different roles in the brain. The former is a component of myelin and plays a critical role in myelin-related functions, ${ }^{49}$ and the latter is involved in axonal growth. ${ }^{50,51}$ Evidence revealed that these lipids can participate in certain 
A

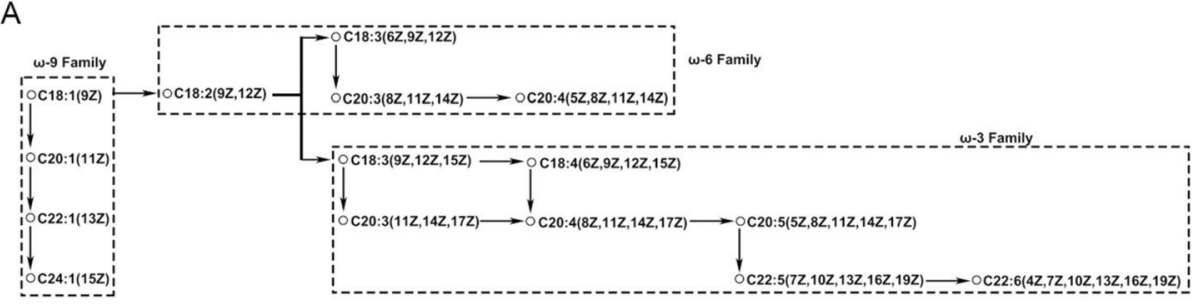

B

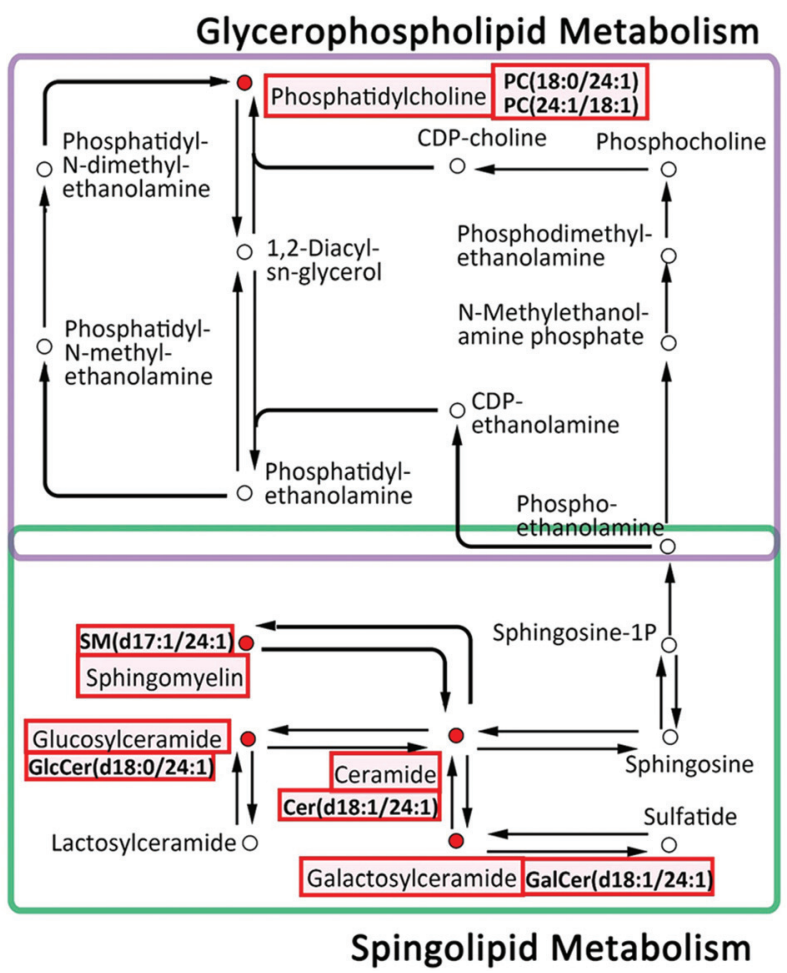

Fig. 8 Biosynthesis of long and very long chain fatty acids in mammals. Biosynthetic pathways of $\omega-3, \omega-6$ and $\omega-9$ family fatty acids (A) and structures and metabolic pathways involved in the conversion of C24:1-lipids in serum and brain (B).

metabolic procedures in brain cells, including structure, ${ }^{52}$ growth, differentiation ${ }^{53}$ and survival, ${ }^{54}$ and hence they affect memory and behavior. ${ }^{53,55,56}$ The previous studies and KEGG pathway analysis of C24:1 $\omega-9$ in the serum and brain revealed that the conversion of $\mathrm{C} 24: 1 \omega-9$ biomarkers can result in sphingolipid metabolism and glycerophospholipid metabolism. C24:1 1 -9 intake together with ASO was accessed firstly in the serum and detected as Cer(d18:1/24:1) and $\operatorname{SM}(\mathrm{d} 17: 1 /$ $24: 1$ ), and then converted and transported to the brain as PC (18:0/24:1), PC(24:1/18:1), GlcCer(d18:0/24:1) and GalCer (d18:1/24:1). The probable conversion pathways according to the previous studies and KEGG pathway analysis of C24:1 $\omega^{-9}$ in the serum and brain are illustrated in Fig. 8B. Previous reports showed that sphingomyelin biosynthesis in mammalian cells is catalyzed by sphingomyelin synthase, which occurs primarily through the transfer of the phosphorylcholine group from phosphatidylcholine to ceramide. ${ }^{57}$ Consequently, sphingomyelin can be degraded to ceramide under the catalysis of acid sphingomyelinase. ${ }^{58}$ Ceramide can galactosylate with the effect of GalCerase to produce galactosylceramide. ${ }^{59}$ These pathways result in sphingolipid metabolism. ${ }^{58}$ Besides, ceramide can be converted into sphingosine due to the effect of ceramidase, and subsequently sphingosine into sphingosine 1-phosphate (S1P) by catalysis with sphingosine kinase. ${ }^{60} \mathrm{~S} 1 \mathrm{P}$ is catalyzed by S1P lyase to form hexadecenal and phosphoethanolamine, which is also included in glycerophospholipid metabolism in the KEGG pathway analysis. Phosphoethanolamine can undergo catalysis by phosphoethanolamine cytidyltransferase and phosphatidylethanolamine $N$-methyltransferase, and eventually convert into phosphatidylcholine. ${ }^{61}$ These pathways may contribute to the conversion of C24:1 $\omega-9$ in the serum and brain and provide the learning and improvement in cognitive function in rats.

Understanding metabolite changes after ASO supplementation can contribute to determining the metabolites related to the improvement in cognitive function and discovering the 
molecular pathways regulated by ASO. The KEGG pathway analysis revealed that the neurotrophin signaling pathway, glycerophospholipid metabolism, sphingolipid metabolism, fat digestion and absorption, glycerolipid metabolism and serotonergic synapse were consecutively and collectively perturbed in the serum and brain (Tables 2 and 3, and Fig. 4). Neurotrophins are a family of trophic factors and considered to play a wide range of roles in the development and function of the nervous system. Certain researches have confirmed that the neurotrophin signaling pathway plays an important role in neural development and additional higher-order activities such as learning and memory. ${ }^{62}$ Glycerophospholipids consist of neuron membranes and play both structural and functional roles. ${ }^{63}$ The stage of well-pronounced Alzheimer's disease-like pathology in OXYS rats is also related to abnormalities in glycerophospholipid metabolism. ${ }^{64}$ Sphingolipids are particularly abundant in the brain and are essential for the development and maintenance of the functional integrity of the nervous system, ${ }^{65}$ and dysregulation of sphingolipid metabolism has been associated with a vast number of neurological diseases via disturbances in membrane organization ${ }^{66}$ such as Alzheimer's disease, Parkinson's disease, several types of epilepsy and Huntington's disease. ${ }^{67}$ Meanwhile, glycerolipid metabolism is associated with neuronal axon regeneration. ${ }^{68}$ Serotonin is a monoamine neurotransmitter and plays an important role. Serotonergic synapse exerts a profound impact on physiological functions including learning and memory, emotion, sleep, pain and motor function, as well as on pathological states such as abnormal mood and cognition. ${ }^{69}$ Theses perturbed pathways indicate that the supplementation of ASO mediated the pathways in FAs metabolism and cognitive improvement.

We found that the improvement in cognitive function was the result of the overall effect of essential fatty acids including NA. Considering that lipid changes and metabolic biomarkers associated with $\omega$-9 fatty acid supplementation have not been reported, our original research revealed overall lipid remodeling and cognitive improvement associated with $\omega-9$ fatty acid supplementation, especially NA supplementation, through serum and whole brain analyses in healthy mice. The contribution of this study is to provide the basis of metabolic theory and methodology for the future application of $\omega-9$ fatty acid or compositions. Based on the results of the metabolic characteristics of the $\omega-9$ fatty acid and lipid remodeling, we can design accurate disease model rat experiments in the future and conduct more detailed brain segmentation studies (such as white matter in hippocampus). The half-life of NA in the serum and RBC of NA is an important experiment for the clinical application of single lipids, and we will use separate compounds to supplement and conduct follow-up studies in the future.

\section{Author contributions}

$\mathrm{XY}$ Chen conceptualized and designed the study. T Xue performed the data curation. WT Song, JR Han, FD Peng, CG Ding, F Lin, JJ Li, and JW Gan performed Investigation. XY Chen per- formed the Supervision. WT Song performed the visualization, statistical analysis work and drafted the manuscript, and WT Song, K Zhang, and SFT Agassi helped edit and revise the paper. All authors read and approved the final manuscript.

\section{Conflicts of interest}

There are no conflicts to declare.

\section{Acknowledgements}

This work was supported by the Fujian Sanming Science and Technology Plan Project: Nervonic acid interferes with cognitive function in Alzheimer's disease [grant numbers no: 2019-S-3], and Fujian Provincial Science and Technology Project: Nervonic acid intervenes in the process of Alzheimer's disease by regulating the intestinal flora and metabolism [grant number 2020J011271].

\section{References}

1 A. A. Spector and H. Y. Kim, Discovery of essential fatty acids, J. Lipid Res., 2015, 56, 11-21.

2 J. Whelan and K. Fritsche, Linoleic acid, Adv. Nutr., 2013, 4, 311-312.

3 G. P. Amminger, M. R. Schafer, C. M. Klier, J. M. Slavik, I. Holzer, M. Holub, S. Goldstone, T. J. Whitford, P. D. McGorry and M. Berk, Decreased nervonic acid levels in erythrocyte membranes predict psychosis in helpseeking ultra-high-risk individuals, Mol. Psychiatry, 2012, 17, 1150-1152.

4 A. J. Richardson and B. K. Puri, The potential role of fatty acids in attention-deficit/hyperactivity disorder, Prostaglandins, Leukotrienes Essent. Fatty Acids, 2000, 63, 79-87.

5 F. Babin, P. Sarda, B. Limasset, B. Descomps, D. Rieu, F. Mendy and A. Crastes de Paulet, Nervonic acid in red blood cell sphingomyelin in premature infants: an index of myelin maturation?, Lipids, 1993, 28, 627-630.

6 C. L. Kien, J. Y. Bunn, C. L. Tompkins, J. A. Dumas, K. I. Crain, D. B. Ebenstein, T. R. Koves and D. M. Muoio, Substituting dietary monounsaturated fat for saturated fat is associated with increased daily physical activity and resting energy expenditure and with changes in mood, Am. J. Clin. Nutr., 2013, 97, 689-697.

7 W. B. Rizzo, R. T. Leshner, A. Odone, A. L. Dammann, D. A. Craft, M. E. Jensen, S. S. Jennings, S. Davis, R. Jaitly and J. A. Sgro, Dietary erucic acid therapy for X-linked adrenoleukodystrophy, Neurology, 1989, 39, 1415-1422.

8 L. Li, W. J. Manning, L. Tong and X. Wang, Chronic drought stress reduced but not protected Shantung maple (Acer truncatum Bunge) from adverse effects of ozone (O3) on growth and physiology in the suburb of Beijing, China, Environ. Pollut., 2015, 201, 34-41. 
9 W. H. Zhao, J. F. Zhang, W. Zhe, Y. X. Zhang and W. X. Tian, The extract of leaves of Acer truncatum Bunge: A natural inhibitor of fatty acid synthase with antitumor activity, J. Enzyme Inhib. Med. Chem., 2006, 21, 589-596.

10 D. J. Chen, L. H. Yan, Q. Li, C. J. Zhang, C. L. Si, Z. Y. Li, Y. J. Song, H. Zhou, T. C. Zhang and X. G. Luo, Bioconversion of conjugated linoleic acid by Lactobacillus plantarum CGMCC8198 supplemented with Acer truncatum bunge seeds oil, Food Sci. Biotechnol., 2017, 26, 15951611.

11 Q. Ma, Y. Wang, L. Zhu, C. Bi, S. Li, S. Li, J. Wen, K. Yan and Q. Li, Characterization of the Complete Chloroplast Genome of Acer truncatum Bunge (Sapindales: Aceraceae): A New Woody Oil Tree Species Producing Nervonic Acid, BioMed Res. Int., 2019, 2019, 7417239.

12 X. Y. Wang, Fan J. S., S. Y. Wang and R. C. Sun, A new resource of nervonic acid from purpleblow maple (Acer truncatum) seed oil, For. Prod. J., 2006, 56(11-12), 147-150.

13 N. Lewkowicz, P. Piatek, M. Namiecinska, M. Domowicz, R. Bonikowski, J. Szemraj, P. Przygodzka, M. Stasiolek and P. Lewkowicz, Naturally Occurring Nervonic Acid Ester Improves Myelin Synthesis by Human Oligodendrocytes, Cells, 2019, 8(8), 786.

14 S. Ding, Y. Gu, Y. Cai, M. Cai, T. Yang, S. Bao, W. Shen, X. Ni, G. Chen and L. Xing, Integrative systems and functional analyses reveal a role of dopaminergic signaling in myelin pathogenesis, J. Transl. Med., 2020, 18, 109.

15 H. Haas, C. L. Oliveira, I. L. Torriani, E. Polverini, A. Fasano, G. Carlone, P. Cavatorta and P. Riccio, Small angle X-ray scattering from lipid-bound myelin basic protein in solution, Biophys. J., 2004, 86, 455-460.

16 M. Martinez and I. Mougan, Fatty acid composition of human brain phospholipids during normal development, J. Neurochem., 1998, 71, 2528-2533.

17 E. Chivandi, B. C. Davidson and K. H. Erlwanger, A comparison of the lipid and fatty acid profiles from the kernels of the fruit (nuts) of Ximenia caffra and Ricinodendron rautanenii from Zimbabwe, Ind. Crops Prod., 2008, 27, 29-32.

18 Q. Li, J. Chen, X. Yu and J. M. Gao, A mini review of nervonic acid: Source, production, and biological functions, Food Chem., 2019, 301, 125286.

19 C. Cook, J. Barnett, K. Coupland and J. Sargent, Effects of feeding Lunaria oil rich in nervonic and erucic acids on the fatty acid compositions of sphingomyelins from erythrocytes, liver, and brain of the quaking mouse mutant, Lipids, 1998, 33, 993-1000.

20 R. Yang, L. Zhang, P. Li, L. Yu and Q. Zhang, A review of chemical composition and nutritional properties of minor vegetable oils in China, Trends Food Sci. Technol., 2018, 74, 26-32.

21 S. S. Patil, B. Sunyer, H. Hoger and G. Lubec, Evaluation of spatial memory of $\mathrm{C} 57 \mathrm{BL} / 6 \mathrm{~J}$ and CD1 mice in the Barnes maze, the Multiple T-maze and in the Morris water maze, Behav. Brain Res., 2009, 198, 58-68.

22 M. M. Zhou, H. X. Che, J. Q. Huang, T. T. Zhang, J. Xu, C. H. Xue and Y. M. Wang, Comparative Study of Different
Polar Groups of EPA-Enriched Phospholipids on Ameliorating Memory Loss and Cognitive Deficiency in Aged SAMP8 Mice, Mol. Nutr. Food Res., 2018, 62, e1700637.

23 M. H. Sarafian, M. Gaudin, M. R. Lewis, F. P. Martin, E. Holmes, J. K. Nicholson and M. E. Dumas, Objective set of criteria for optimization of sample preparation procedures for ultra-high throughput untargeted blood plasma lipid profiling by ultra performance liquid chromatography-mass spectrometry, Anal. Chem., 2014, 86, 57665774.

24 H. Zhong, C. Fang, Y. Fan, Y. Lu, B. Wen, H. Ren, G. Hou, F. Yang, H. Xie, Z. Jie, Y. Peng, Z. Ye, J. Wu, J. Zi, G. Zhao, J. Chen, X. Bao, Y. Hu, Y. Gao, J. Zhang, H. Yang, J. Wang, L. Madsen, K. Kristiansen, C. Ni, J. Li and S. Liu, Lipidomic profiling reveals distinct differences in plasma lipid composition in healthy, prediabetic, and type 2 diabetic individuals, GigaScience, 2017, 6, 1-12.

25 S. E. Wahrle, H. Jiang, M. Parsadanian, J. Legleiter, X. Han, J. D. Fryer, T. Kowalewski and D. M. Holtzman, ABCA1 is required for normal central nervous system ApoE levels and for lipidation of astrocyte-secreted apoE, J. Biol. Chem., 2004, 279, 40987-40993.

26 B. Karten, R. B. Campenot, D. E. Vance and J. E. Vance, Expression of ABCG1, but not ABCA1, correlates with cholesterol release by cerebellar astroglia, J. Biol. Chem., 2006, 281, 4049-4057.

27 N. Camargo, A. Goudriaan, A. F. van Deijk, W. M. Otte, J. F. Brouwers, H. Lodder, D. H. Gutmann, K. A. Nave, R. M. Dijkhuizen, H. D. Mansvelder, R. Chrast, A. B. Smit and M. H. G. Verheijen, Oligodendroglial myelination requires astrocyte-derived lipids, PLoS Biol., 2017, 15, e1002605.

28 A. V. Witte, L. Kerti, H. M. Hermannstadter, J. B. Fiebach, S. J. Schreiber, J. P. Schuchardt, A. Hahn and A. Floel, Long-chain omega-3 fatty acids improve brain function and structure in older adults, Cereb. Cortex, 2014, 24, 30593068.

29 C. E. Hayes and J. M. Ntambi, Multiple Sclerosis: Lipids, Lymphocytes, and Vitamin D, Immunometabolism, 2020, 2(3), e200019.

30 S. Aggarwal, L. Yurlova and M. Simons, Central nervous system myelin: structure, synthesis and assembly, Trends Cell Biol., 2011, 21, 585-593.

31 B. Gerstl, M. G. Tavaststjerna, L. F. Eng and J. K. Smith, Sphingolipids and their precursors in human brain (normal and MS), Z. Neurol., 1972, 202, 104-120.

32 J. R. Sargent, K. Coupland and R. Wilson, Nervonic acid and demyelinating disease, Med. Hypotheses, 1994, 42, 237242.

33 T. C. Kuo and Y. J. Tseng, LipidPedia: a comprehensive lipid knowledgebase, Bioinformatics, 2018, 34, 2982-2987.

34 I. Alecu and S. A. L. Bennett, Dysregulated Lipid Metabolism and Its Role in alpha-Synucleinopathy in Parkinson's Disease, Front. Neurosci., 2019, 13, 328.

35 C. P. Muller, M. Reichel, C. Muhle, C. Rhein, E. Gulbins and J. Kornhuber, Brain membrane lipids in major 
depression and anxiety disorders, Biochim. Biophys. Acta, 2015, 1851, 1052-1065.

36 P. Aubourg, C. Adamsbaum, M. C. Lavallard-Rousseau, F. Rocchiccioli, N. Cartier, I. Jambaque, C. Jakobezak, A. Lemaitre, F. Boureau, C. Wolf, et al., A two-year trial of oleic and erucic acids ("Lorenzo's oil") as treatment for adrenomyeloneuropathy, N. Engl. J. Med., 1993, 329, 745752.

37 I. Galan-Arriero, D. Serrano-Munoz, J. Gomez-Soriano, C. Goicoechea, J. Taylor, A. Velasco and G. Avila-Martin, The role of Omega-3 and Omega-9 fatty acids for the treatment of neuropathic pain after neurotrauma, Biochim. Biophys. Acta, Biomembr., 2017, 1859, 1629-1635.

38 E. Kim, H. J. Ko, S. J. Jeon, S. Lee, H. E. Lee, H. N. Kim, E. R. Woo and J. H. Ryu, The memory-enhancing effect of erucic acid on scopolamine-induced cognitive impairment in mice, Pharmacol., Biochem. Behav., 2016, 142, 85-90.

39 J. Assies, R. Lieverse, P. Vreken, R. J. Wanders, P. M. Dingemans and D. H. Linszen, Significantly reduced docosahexaenoic and docosapentaenoic acid concentrations in erythrocyte membranes from schizophrenic patients compared with a carefully matched control group, Biol. Psychiatry, 2001, 49, 510-522.

40 Z. J. Wang, C. L. Liang, G. M. Li, C. Y. Yu and M. Yin, Neuroprotective effects of arachidonic acid against oxidative stress on rat hippocampal slices, Chem.-Biol. Interact., 2006, 163, 207-217.

41 M. Saito and M. Saito, Incorporation of very-long-chain fatty acids into sphingolipids of cultured neural cells, J. Neurochem., 1991, 57, 465-469.

42 J. B. S. Kumar and B. Sharma, A review on neuropharmacological role of erucic acid: an omega-9 fatty acid from edible oils, Nutr. Neurosci., 2020, 1-15.

43 M. Xiang, G. Alfven, M. Blennow, M. Trygg and R. Zetterstrom, Long-chain polyunsaturated fatty acids in human milk and brain growth during early infancy, Acta Paediatr., 2000, 89, 142-147.

44 J. C. Demar Jr., K. Ma, L. Chang, J. M. Bell and S. I. Rapoport, alpha-Linolenic acid does not contribute appreciably to docosahexaenoic acid within brain phospholipids of adult rats fed a diet enriched in docosahexaenoic acid, J. Neurochem., 2005, 94, 1063-1076.

45 A. H. Merrill Jr., E. M. Schmelz, E. Wang, D. L. Dillehay, L. G. Rice, F. Meredith and R. T. Riley, Importance of sphingolipids and inhibitors of sphingolipid metabolism as components of animal diets, J. Nutr., 1997, 127, 830S833S.

46 W. S. Kim, J. H. Hsiao, S. Bhatia, E. N. Glaros, A. S. Don, S. Tsuruoka, C. Shannon Weickert and G. M. Halliday, ABCA8 stimulates sphingomyelin production in oligodendrocytes, Biochem. J., 2013, 452, 401-410.

47 D. K. Perry and Y. A. Hannun, The role of ceramide in cell signaling, Biochim. Biophys. Acta, 1998, 1436, 233-243.

48 D. J. Canty and S. H. Zeisel, Lecithin and choline in human health and disease, Nutr. Rev., 1994, 52, 327-339.
49 T. Coetzee, N. Fujita, J. Dupree, R. Shi, A. Blight, K. Suzuki and B. Popko, Myelination in the absence of galactocerebroside and sulfatide: normal structure with abnormal function and regional instability, Cell, 1996, 86, 209-219.

50 S. Watanabe, S. Endo, E. Oshima, T. Hoshi, H. Higashi, K. Yamada, K. Tohyama, T. Yamashita and Y. Hirabayashi, Glycosphingolipid synthesis in cerebellar Purkinje neurons: roles in myelin formation and axonal homeostasis, Glia, 2010, 58, 1197-1207.

51 A. Schwarz and A. H. Futerman, Distinct roles for ceramide and glucosylceramide at different stages of neuronal growth, J. Neurosci., 1997, 17, 2929-2938.

52 M. Eckhardt, The role and metabolism of sulfatide in the nervous system, Mol. Neurobiol., 2008, 37, 93-103.

53 H. Rahmann, Brain gangliosides and memory formation, Behav. Brain Res., 1995, 66, 105-116.

54 R. G. Cutler and M. P. Mattson, Sphingomyelin and ceramide as regulators of development and lifespan, Mech. Ageing Dev., 2001, 122, 895-908.

55 E. Gulbins, M. Palmada, M. Reichel, A. Luth, C. Bohmer, D. Amato, C. P. Muller, C. H. Tischbirek, T. W. Groemer, G. Tabatabai, K. A. Becker, P. Tripal, S. Staedtler, T. F. Ackermann, J. van Brederode, C. Alzheimer, M. Weller, U. E. Lang, B. Kleuser, H. Grassme and J. Kornhuber, Acid sphingomyelinase-ceramide system mediates effects of antidepressant drugs, Nat. Med., 2013, 19, 934-938.

56 S. Y. Chung, T. Moriyama, E. Uezu, K. Uezu, R. Hirata, N. Yohena, Y. Masuda, T. Kokubu and S. Yamamoto, Administration of phosphatidylcholine increases brain acetylcholine concentration and improves memory in mice with dementia, J. Nutr., 1995, 125, 1484-1489.

57 L. Riboni, P. Viani, R. Bassi, P. Giussani and G. Tettamanti, Basic fibroblast growth factor-induced proliferation of primary astrocytes. evidence for the involvement of sphingomyelin biosynthesis, J. Biol. Chem., 2001, 276, 1279712804.

58 G. van Echten-Deckert and S. Alam, Sphingolipid metabolism - an ambiguous regulator of autophagy in the brain, Biol. Chem., 2018, 399, 837-850.

59 D. Russo, L. Capolupo, J. S. Loomba, L. Sticco and G. D'Angelo, Glycosphingolipid metabolism in cell fate specification, J. Cell Sci., 2018, 131(24), jcs219204.

60 R. Romero-Guevara, F. Cencetti, C. Donati and P. Bruni, Sphingosine 1-phosphate signaling pathway in inner ear biology. New therapeutic strategies for hearing loss?, Front. Aging Neurosci., 2015, 7, 60.

61 A. Kihara, Sphingosine 1-phosphate is a key metabolite linking sphingolipids to glycerophospholipids, Biochim. Biophys. Acta, 2014, 1841, 766-772.

62 L. F. Reichardt, Neurotrophin-regulated signalling pathways, Philos. Trans. R. Soc., B, 2006, 361, 1545-1564.

$63 \mathrm{H}$. Palsdottir and C. Hunte, Lipids in membrane protein structures, Biochim. Biophys. Acta, 2004, 1666, 2-18.

64 N. A. Stefanova, N. I. Ershov and N. G. Kolosova, Suppression of Alzheimer's Disease-Like Pathology Progression by Mitochondria-Targeted Antioxidant SkQ1: A 
Transcriptome Profiling Study, Oxid. Med. Cell. Longevity, 2019, 2019, 3984906.

65 G. van Echten-Deckert and T. Herget, Sphingolipid metabolism in neural cells, Biochim. Biophys. Acta, 2006, 1758, 1978-1994.

66 M. Piccinini, F. Scandroglio, S. Prioni, B. Buccinna, N. Loberto, M. Aureli, V. Chigorno, E. Lupino, G. DeMarco, A. Lomartire, M. T. Rinaudo, S. Sonnino and A. Prinetti, Deregulated sphingolipid metabolism and membrane organization in neurodegenerative disorders, Mol. Neurobiol., 2010, 41, 314-340.
67 A. S. B. Olsen and N. J. Faergeman, Sphingolipids: membrane microdomains in brain development, function and neurological diseases, Open Biol., 2017, 7, 170069.

68 C. Yang, X. Wang, J. Wang, W. Chen, N. Lu, S. Siniossoglou, Z. Yao and K. Liu, Rewiring Neuronal Glycerolipid Metabolism Determines the Extent of Axon Regeneration, Neuron, 2020, 105, 276-292.

69 M. J. Millan, P. Marin, J. Bockaert and C. Mannoury la Cour, Signaling at G-protein-coupled serotonin receptors: recent advances and future research directions, Trends Pharmacol. Sci., 2008, 29, 454-464. 